Homology, Homotopy and Applications, vol.21(1), 2019, pp.351-373

\title{
CONVOLUTION ALGEBRAS AND THE DEFORMATION THEORY OF INFINITY-MORPHISMS
}

\author{
DANIEL ROBERT-NICOUD AND FELIX WIERSTRA
}

(communicated by Nathalie Wahl)

\begin{abstract}
Given a coalgebra $C$ over a cooperad and an algebra $A$ over an operad, it is often possible to define a natural homotopy Lie algebra structure on $\operatorname{hom}(C, A)$, the space of linear maps between them, called the convolution algebra of $C$ and $A$. In the present article, we use convolution algebras to define the deformation complex for $\infty$-morphisms of algebras over operads and coalgebras over cooperads. We also complete the study of the compatibility between convolution algebras and $\infty$-morphisms of algebras and coalgebras. We prove that the convolution algebra bifunctor can be extended to a bifunctor that accepts $\infty$ morphisms in both slots and which is well defined up to homotopy, and we generalize and take a new point of view on some other already known results. This paper concludes a series of works by the two authors dealing with the investigation of convolution algebras.
\end{abstract}

\section{Contents}

1 Introduction

2 Infinity-morphisms and convolution algebras

3 Compatibility between convolution algebras and infinity-morphisms

4 Proof of Theorems 2.4, 3.1, and 3.6

The first author was supported by grants from Région Ile-de-France, and the grant ANR-14-CE250008-01 project SAT.

The second author acknowledges the financial support from the grant GA CR No. P201/12/G028.

Received June 13, 2018, revised September 2, 2018; published on November 7, 2018.

2010 Mathematics Subject Classification: Primary 18D50; Secondary 08C05, 18G55.

Key words and phrases: Homotopical algebra, convolution algebra, infinity-morphism.

Article available at http://dx.doi.org/10.4310/HHA.2019.v21.n1.a17

Copyright (C) 2018, International Press. Permission to copy for private use granted. 


\section{Introduction}

Suppose that we are given a type of algebra - such as associative, commutative or Lie algebras, but also more elaborate ones, such as homotopy Lie or hypercommutative algebras - and a type of coalgebra - coassociative, cocommutative, and so on - encoded respectively by an operad $\mathscr{P}$ and a cooperad $\mathscr{C}$. Suppose that these types of algebras and coalgebras are related by an operadic twisting morphism $\alpha: \mathscr{C} \rightarrow \mathscr{P}$. Some interesting examples of these include the universal twisting morphisms associated to the operadic bar and cobar constructions, and the twisting morphisms given by Koszul duality. Then, given a $\mathscr{C}$-coalgebra $C$ and a $\mathscr{P}$-algebra $A$, one can equip the chain complex of linear maps $\operatorname{hom}(C, A)$ with the structure of a (shifted) homotopy Lie algebra (usually referred to as $\triangleleft \mathscr{L}_{\infty}$-algebras) in a canonical way. We denote this algebra by $\operatorname{hom}^{\alpha}(C, A)$ and call it the convolution algebra of $C$ and $A$.

These algebras have already found various applications. They helped to construct a "universal Maurer-Cartan element" in [RN17], they are used to construct complete rational invariants of maps between topological spaces in [Wie16], and they were applied to the construction of rational models for mapping spaces in [RNW17].

The first of the two main results of the present paper is that one can use convolution algebras to define the correct deformation complex for $\infty$-morphisms between $\mathscr{P}$-algebras, as well as $\infty$-morphisms between conilpotent $\mathscr{C}$-coalgebras. Namely, given two $\mathscr{P}$-algebras, resp. conilpotent $\mathscr{C}$-coalgebras, we define a shifted homotopy Lie algebra whose Maurer-Cartan elements are in natural bijection with the $\infty$-morphisms between the algebras, resp. coalgebras, and which is such that two Maurer-Cartan elements are gauge equivalent if and only if the corresponding $\infty$ morphisms are homotopic. We also relate the $\infty$-groupoid associated to the deformation complex with the mapping space in the $\infty$-category of algebras. Some partial results in this direction were already given e.g., in [Dol07, DHR15], and [RN18a, Sect. 7.1].

Convolution algebras have been proven to behave well with respect to the tools of homotopical algebra. For example [RN18a, Thm. 5.1], they are compatible with the homotopy transfer theorem, see e.g., [LV12, Sect. 10.3]. They are also compatible with a generalized notion of morphisms, called $\infty$-morphisms, in the sense that the bifunctor $\operatorname{hom}^{\alpha}(-,-)$ can be extended to a bifunctor accepting $\infty$-morphisms in either one of its slots. This was proven in [RN18a, Prop. 4.4] for a special case, and in full generality in [RNW17, Sect. 5.2]. Unfortunately, in op. cit. the authors were also able to prove that one cannot perform the next natural step and extend the two bifunctors to a bifunctor accepting $\infty$-morphisms in both slots. The counterexample consists in an $\infty$-morphism $\Phi$ of $\mathscr{C}$-coalgebras and an $\infty$-morphism $\Psi$ of $\mathscr{P}$-algebras such that the two composites

$$
\operatorname{hom}^{\alpha}(\Phi, 1) \operatorname{hom}^{\alpha}(1, \Psi) \quad \text { and } \quad \operatorname{hom}^{\alpha}(1, \Psi) \operatorname{hom}^{\alpha}(\Phi, 1)
$$

are not equal, which tells us that a common extension to a new bifunctor is impossible.

The second main result of the present article is that, assuming that the twisting morphism $\alpha$ is Koszul, these two composites are homotopic as $\infty$-morphisms of shifted homotopy Lie algebras. In particular, it is possible to extend the bifunctor hom ${ }^{\alpha}(-,-)$ to take $\infty$-morphisms in both slots if one accepts to work only up to homotopy.

The content of this article is as follows. Section 2 begins with a short recollection 
of the less classical background notions we will use: those of convolution algebras and $\infty$-morphisms relative to a twisting morphism. This is followed by giving an interpretation of the Maurer-Cartan elements of a convolution algebra in terms of usual morphisms of algebras and coalgebras, and showing that two such MaurerCartan elements are gauge equivalent if and only if the associated morphisms are homotopic. This is Theorem 2.4, and it motivates the construction of a deformation complex for $\infty$-morphisms between algebras or coalgebras using convolution algebras.

Section 3 contains most of the new results of the present article. The main result of the section is Theorem 3.1, which describes a morphism of $₫ \mathscr{L}_{\infty}$-algebras between certain convolution algebras, and which gives us all the tools we need to study the compatibility of convolution algebras with $\infty$-morphisms. The rest of the section is mostly composed by consequences of this main theorem, and reaches its culmination with Theorem 3.6, which tells us that, even though we cannot extend the bifunctor $\operatorname{hom}^{\alpha}(-,-)$ to a bifunctor taking $\infty$-morphisms in both its slots, we can do so if we pass to the homotopy categories provided the twisting morphism $\alpha$ is Koszul. The precise statement is that the two compositions described in (1) are homotopic.

Throughout the text, we postpone various technical proofs in order to improve readability. These are collected in Section 4. This section also contains a result of independent interest. This is Theorem 4.1, which compares the deformation complex described at the end of Section 2 with another natural construction, proving that they contain exactly the same information. This last result also relates the Maurer-Cartan $\infty$-groupoid of the deformation complex with the $\infty$-categorical mapping space for algebras over an operad.

We conclude the paper with Appendix A, where we give an explicit counterexample to the conclusion of Theorem 3.6 if we remove the assumption that the twisting morphism is Koszul.

This paper concludes a series of articles by the two authors dealing with the investigation of convolution algebras which started with [Wie16], and [RN18a], and then continued jointly with [RNW17].

\section{Acknowledgments}

Both authors are grateful to Bruno Vallette and Alexander Berglund for their comments, advice, and constant support.

\section{Notation and conventions}

We will use essentially the same notation and conventions as in [RNW17]. By transitivity, we will follow the notation of the book [LV12] as closely as possible when talking about operads.

We work over a field $\mathbb{K}$ of characteristic 0 , and over the category of chain complexes. The dual of a chain complex will again be seen as a chain complex. All operads and cooperads in this paper are implicitly assumed to be reduced, meaning that they are zero in arity 0 , and spanned by the identity in arity 1 . Similarly, all coalgebras and cooperads are assumed to be conilpotent.

When talking about the homotopy theory of algebras, we always place ourselves in the Hinich model structure [Hin97, Thm. 4.1.1], where the fibrations are the surjections, and the weak equivalences are the maps of algebras that are quasi-isomorphisms 
of the underlying chain complexes. When considering coalgebras, the model structure we will use is the one defined in [DCH16] generalizing [Val14, Sect. 2.1], and depends on the specific twisting morphism we are working with. If $\alpha: \mathscr{C} \rightarrow \mathscr{P}$ is a twisting morphism, the associated model structure on $\mathscr{C}$-coalgebras has the injections as cofibrations, and the fibrations and weak equivalences are created by the cobar functor $\Omega_{\alpha}$. This means that a morphism of coalgebras $f$ is a fibration, resp. a weak equivalence, if and only if $\Omega_{\alpha} f$ is surjective, resp. a quasi-isomorphism. All Koszul morphisms induce the same model structure by [LG16, Prop. 32]. In the Koszul case, the class of weak equivalences is the closure of the class of filtered quasi-isomorphisms of coalgebras under the 2-out-of-3 property, see [RN18b, Thm. 4.9].

\section{Infinity-morphisms and convolution algebras}

Since this paper is a follow-up of the article [RNW17], we will keep the recollections to a minimum and we refer the reader to op. cit. for any need of reminders on the topic of convolution algebras or the theory surrounding them. We give nonetheless a small list of definitions and basic facts that we will need throughout the paper, and give an upgraded version of [Wie16, Thm. 7.1], see Theorem 2.4.

\subsection{Infinity-morphisms relative to a twisting morphism}

The notions of $\infty$-morphisms of algebras and coalgebras relative to a twisting morphism are defined as follows.

Definition 2.1. Let $\mathscr{C}$ be a cooperad, let $\mathscr{P}$ be an operad, and let $\alpha: \mathscr{C} \rightarrow \mathscr{P}$ be a twisting morphism.

1. Let $A, A^{\prime}$ be two $\mathscr{P}$-algebras. An $\infty$-morphism $\Psi$ of $\mathscr{P}$-algebras relative to $\alpha$ - or an $\infty_{\alpha}$-morphism - from $A$ to $A^{\prime}$ is a morphism

$$
\Psi: \mathrm{B}_{\alpha} A \longrightarrow \mathrm{B}_{\alpha} A^{\prime}
$$

of $\mathscr{C}$-coalgebras. We also write $\Psi: A \rightsquigarrow A^{\prime}$.

2. Let $C^{\prime}, C$ be two $\mathscr{C}$-coalgebras. An $\infty$-morphism $\Phi$ of $\mathscr{C}$-coalgebras relative to $\alpha$ - or an $\infty_{\alpha}$-morphism - from $C^{\prime}$ to $C$ is a morphism

$$
\Psi: \Omega_{\alpha} C^{\prime} \longrightarrow \Omega_{\alpha} C
$$

of $\mathscr{P}$-algebras. We also write $\Phi: C^{\prime} \rightsquigarrow C$.

These notions of $\infty$-morphisms relative to a twisting morphism were studied in [RNW17, Sect. 3].

We know that for any twisting morphism $\alpha: \mathscr{C} \rightarrow \mathscr{P}$ the relative bar construction $\mathrm{B}_{\alpha}$ preserves fibrations, and dually the relative cobar construction $\Omega_{\alpha}$ preserves cofibrations, as they form a Quillen pair (see [DCH16, Thm. 3.11(1)], and [Val14, Thm. $2.9]$ for the Koszul case). Therefore, any coalgebra of the form $\mathrm{B}_{\alpha} A$ is fibrant, and any algebra of the form $\Omega_{\alpha} C$ is cofibrant. We also know that all $\mathscr{P}$-algebras are fibrant, and that all $\mathscr{C}$-coalgebras are cofibrant. Therefore, we can see $\infty_{\alpha}$-morphisms, both of algebras and coalgebras, as morphisms between bifibrant objects. In particular, the homotopy relation is an equivalence relation for them.

Definition 2.2. Let $\alpha: \mathscr{C} \rightarrow \mathscr{P}$ be a twisting morphism. 
1. Two $\infty_{\alpha}$-morphisms $A \rightsquigarrow A^{\prime}$ of $\mathscr{P}$-algebras are homotopic (as $\infty_{\alpha}$-morphisms) if they are homotopic seen as morphisms of $\mathscr{C}$-coalgebras $\mathrm{B}_{\alpha} A \rightarrow \mathrm{B}_{\alpha} A^{\prime}$.

2. Dually, two $\infty_{\alpha}$-morphisms $C^{\prime} \rightsquigarrow C$ of $\mathscr{C}$-coalgebras are homotopic (as $\infty_{\alpha^{-}}$ morphisms) if they are homotopic seen as morphisms of $\mathscr{P}$-algebras $\Omega_{\alpha} C^{\prime} \rightarrow$ $\Omega_{\alpha} C$.

One should also compare this notion of homotopy with the results of the article [DHR15].

\subsection{Convolution algebras}

The main subject of interest of this article are convolution algebras and their homotopical properties. We give a short reminder of how these objects appear. Recall that, given a cooperad $\mathscr{C}$ and an operad $\mathscr{P}$, there is a natural operad structure on $\operatorname{hom}(\mathscr{C}, \mathscr{P})$, called the convolution operad. It was introduced in [BM03, Sect. 1], see also [LV12, Sect. 6.4.1]. We denote by $\mathfrak{L}_{\infty}:=\Omega \mathrm{Com}^{\vee}$ the operad encoding shifted homotopy Lie algebras, see e.g., [RNW17, Sect. 2.7].

Theorem 2.3 ([Wie16, Sect. 7]). Let $\mathscr{C}$ be a cooperad, and let $\mathscr{P}$ be an operad. There is a natural, canonical bijection

$$
\left.\operatorname{Tw}(\mathscr{C}, \mathscr{P}) \cong \operatorname{hom}_{\mathrm{Op}}(\lrcorner \mathscr{L}_{\infty}, \operatorname{hom}(\mathscr{C}, \mathscr{P})\right)
$$

between the set of twisting morphisms from $\mathscr{C}$ to $\mathscr{P}$ and the set of morphisms of operads from the operad $₫ \mathscr{L}_{\infty}$ encoding shifted homotopy Lie algebras to the convolution operad $\operatorname{hom}(\mathscr{C}, \mathscr{P})$.

This bijection is explicitly given by sending a twisting morphism $\alpha: \mathscr{C} \rightarrow \mathscr{P}$ to

$$
\mathrm{W}_{\alpha}: \diamond \mathscr{L}_{\infty} \longrightarrow \operatorname{hom}(\mathscr{C}, \mathscr{P})
$$

defined by $W_{\alpha}\left(\mu_{n}^{\vee}\right)=\alpha(n): \mathscr{C}(n) \rightarrow \mathscr{P}(n)$. This assignment is also compatible with morphisms of operads. We refer the reader to [RNW17, Thm. 4.1] for the details.

Let $\alpha: \mathscr{C} \rightarrow \mathscr{P}$ be a twisting morphism, let $C$ be a $\mathscr{C}$-coalgebra, and let $A$ be a $\mathscr{P}$-algebra. Then $\operatorname{hom}(C, A)$ is naturally a $\operatorname{hom}(\mathscr{C}, \mathscr{P})$-algebra, so that we can pull its structure back along $W_{\alpha}$ to get an $\lrcorner \mathscr{L}_{\infty}$-algebra, which we denote by $\operatorname{hom}^{\alpha}(C, A)$ and call the convolution algebra of $C$ and $A$. Explicitly, if $C$ is a $\mathscr{C}$-coalgebra and $A$ is a $\mathscr{P}$-algebra, and denoting $\Delta_{C}: C \rightarrow \mathscr{C}(C)$ and $\gamma_{A}: \mathscr{P}(A) \rightarrow A$ the structure maps of $C$ and $A$ respectively, then the $\lrcorner \mathscr{L}_{\infty}$-algebra structure of $\operatorname{hom}^{\alpha}(C, A)$ is given by

$$
\ell_{n}\left(f_{1}, \ldots, f_{n}\right)=\gamma_{A}(\alpha \otimes F)^{\mathbb{S}} \Delta_{C}^{n},
$$

where $\Delta_{C}^{n}$ is the part of $\Delta_{C}$ landing in $\left(\mathscr{C}(n) \otimes C^{\otimes n}\right)^{\mathbb{S}_{n}}$, and where

$$
(\alpha \otimes F)^{\mathbb{S}}:=\sum_{\sigma \in \mathbb{S}_{n}}(-1)^{\sigma(F)} \alpha \otimes f_{\sigma(1)} \otimes \cdots \otimes f_{\sigma(n)}
$$

for $f_{1}, \ldots, f_{n} \in \operatorname{hom}(C, A)$ and $\sigma(F)$ is the Koszul sign coming from switching around the $f_{i}$. The map $(\alpha \otimes F)^{\mathbb{S}}$ maps from invariants to invariants. Notice that there is an implicit identification of invariants with coinvariants before composing in $A$.

The operation sending $(C, A)$ to $\operatorname{hom}^{\alpha}(C, A)$ is compatible with morphisms of $\mathscr{C}$ coalgebras in the first slot, and with morphisms of $\mathscr{P}$-algebras in the second slot. 
Therefore, we obtain a bifunctor

$$
\left.\operatorname{hom}^{\alpha}:(\mathscr{C} \text {-cog })^{\text {op }} \times \mathscr{P} \text {-alg } \longrightarrow\right\lrcorner \mathscr{L}_{\infty} \text {-alg },
$$

which is given by hom ${ }^{\alpha}(C, A)$ on objects. Here, $\mathscr{C}-\operatorname{cog}$ denotes the category of conilpotent $\mathscr{C}$-coalgebras, and $\mathscr{P}$-alg denotes the category of $\mathscr{P}$-algebras, both with strict morphisms.

\subsection{Maurer-Cartan elements and the deformation complex for infinity- morphisms}

Given an $\lrcorner \mathscr{L}_{\infty}$-algebra, it is natural - from a deformation theoretical point of view - to ask what its Maurer-Cartan elements and gauge relations are. In the case of convolution algebras, we can give a clean and complete answer.

Theorem 2.4. Let $\alpha: \mathscr{C} \rightarrow \mathscr{P}$ be a twisting morphism, let $C$ be a $\mathscr{C}$-algebra, and let $A$ be a $\mathscr{P}$-algebra. Then there are natural bijections

$$
\operatorname{hom}_{\mathscr{C}-\operatorname{cog}}\left(C, \mathrm{~B}_{\alpha} A\right) \cong \mathrm{MC}\left(\operatorname{hom}^{\alpha}(C, A)\right) \cong \operatorname{hom}_{\mathscr{P}} \text {-alg }\left(\Omega_{\alpha} C, A\right) .
$$

Moreover,

1. two morphisms of $\mathscr{C}$-coalgebras $C \rightarrow \mathrm{B}_{\alpha} A$ are homotopic if and only if the respective Maurer-Cartan elements are gauge equivalent, and

2. two morphisms of $\mathscr{P}$-algebras $\Omega_{\alpha} C \rightarrow A$ are homotopic if and only if the respective Maurer-Cartan elements are gauge equivalent.

The proof of this result is postponed to Section 4.1.

Remark 2.5. The Maurer-Cartan equation in the result above is well defined, since all $\mathscr{C}$-coalgebras are supposed to be conilpotent.

Remark 2.6. The characterization of the Maurer-Cartan set of convolution algebras was initially done in [Wie16, Thm. 7.1], where point (1) is also stated, and in [RN18a, Thm. 6.3]. A special case of this result can also be found in [DP16, Thm. 1].

We can use Theorem 2.4 to solve the problem of giving the correct deformation complex for both $\infty$-morphisms between $\mathscr{P}$-algebras, and $\infty$-morphisms between $\mathscr{C}$ coalgebras. The problem of defining such a deformation complex was mentioned by M. Kontsevich in his 2017 talk at Séminaire Bourbaki [Kon17]. A first approach to its solution was given by [RN18a, Thm. 7.1].

Definition 2.7. Let $\alpha: \mathscr{C} \rightarrow \mathscr{P}$ be a twisting morphism.

1. Let $A, A^{\prime}$ be two $\mathscr{P}$-algebras. The deformation complex of $\infty_{\alpha}$-morphisms of $\mathscr{P}$-algebras from $A$ to $A^{\prime}$ is the $\lrcorner \mathscr{L}_{\infty}$-algebra $\operatorname{hom}^{\alpha}\left(\mathrm{B}_{\alpha} A, A^{\prime}\right)$.

2. Let $C^{\prime}, C$ be two $\mathscr{C}$-coalgebras. The deformation complex of $\infty_{\alpha}$-morphisms of $\mathscr{C}$-coalgebras from $C^{\prime}$ to $C$ is the $s \mathscr{L}_{\infty}$-algebra $\operatorname{hom}^{\alpha}\left(C^{\prime}, \Omega_{\alpha} C\right)$.

Indeed, a Maurer-Cartan element of $\operatorname{hom}^{\alpha}\left(\mathrm{B}_{\alpha} A, A^{\prime}\right)$ is the same thing as a morphism $\mathrm{B}_{\alpha} A \rightarrow \mathrm{B}_{\alpha} A^{\prime}$, i.e., an $\infty_{\alpha}$-morphism $A \rightsquigarrow A^{\prime}$. Moreover, being homotopic as morphisms of $\mathscr{C}$-coalgebras gives an equivalence relation between $\infty_{\alpha}$-morphisms of $\mathscr{P}$-algebras, as the bar construction $\mathrm{B}_{\alpha}$ lands in the bifibrant $\mathscr{C}$-coalgebras. In [Val14, 
Sect. 3.2], it was shown that if $\alpha$ is Koszul, then this is the correct notion of homotopy equivalence for $\infty_{\alpha}$-morphisms. Dually, a Maurer-Cartan element of hom ${ }^{\alpha}\left(C^{\prime}, \Omega_{\alpha} C\right)$ is the same thing as an $\infty_{\alpha}$-morphism $C^{\prime} \rightsquigarrow C$ of $\mathscr{C}$-coalgebras, and being homotopic as morphisms of $\mathscr{P}$-algebras is an equivalence relation on these morphisms.

\section{Compatibility between convolution algebras and infinity-morphisms}

The main result of this section is the fact that certain natural maps between certain deformation complexes are morphisms of $₫ \mathscr{L}_{\infty}$-algebras. It has many interesting and important consequences, which we explore in Sections 3.2 and 3.3. In particular, we recover the two bifunctors from $\left[R N W 17\right.$, Sect. 5] extending $\operatorname{hom}^{\alpha}(-,-)$, and we prove that they commute in the homotopy category of $\lrcorner \mathscr{L}_{\infty}$-algebras and their $\infty$ morphisms.

\subsection{Statement of the main theorem}

Fix a twisting morphism $\alpha: \mathscr{C} \rightarrow \mathscr{P}$, and let $A, A^{\prime}$ be two $\mathscr{P}$-algebras. Suppose we are given

$$
x \in \operatorname{hom}^{\alpha}\left(\mathrm{B}_{\alpha} A, A^{\prime}\right) .
$$

Then given any $\mathscr{C}$-coalgebra $C$, we define a map

$$
\operatorname{hom}_{r}^{\alpha}(1, x): \mathrm{B}_{\iota} \operatorname{hom}^{\alpha}(C, A) \longrightarrow \operatorname{hom}^{\alpha}\left(C, A^{\prime}\right),
$$

where $\iota: \mathrm{Com}^{\vee} \rightarrow \Omega \mathrm{Com}^{\vee}=\triangleleft \mathscr{L}_{\infty}$ is the natural twisting morphism. It is given as follows. Let $f_{1}, \ldots, f_{n} \in \operatorname{hom}(C, A)$, and let $F:=f_{1} \otimes \cdots \otimes f_{n}$ for brevity. Similarly to what was done before, denote by

$$
F^{\mathbb{S}}:\left(\mathscr{C}(n) \otimes C^{\otimes n}\right)^{\mathbb{S}_{n}} \longrightarrow\left(\mathscr{C}(n) \otimes A^{\otimes n}\right)^{\mathbb{S}_{n}}
$$

the map

$$
F^{\mathbb{S}}:=\sum_{\sigma \in \mathbb{S}_{n}}(-1)^{\sigma(F)} \mathrm{id}_{\mathscr{C}} \otimes f_{\sigma(1)} \otimes \cdots \otimes f_{\sigma(n)},
$$

where $\sigma(F)$ is the Koszul sign obtained by switching around the $f_{i}$. We define $\operatorname{hom}_{r}^{\alpha}(1, x)$ by the following diagram:

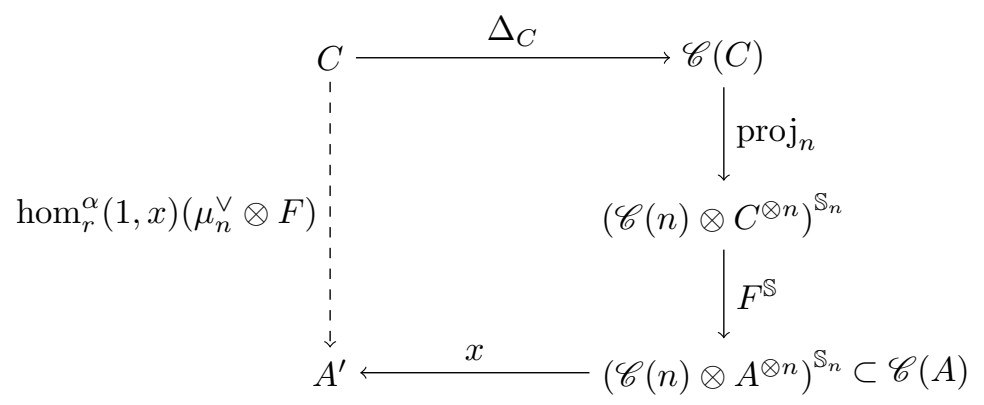


Dually, let $C^{\prime}, C$ be two $\mathscr{C}$-coalgebras, and suppose we have $y \in \operatorname{hom}^{\alpha}\left(C^{\prime}, \Omega_{\alpha} C\right)$. Given a $\mathscr{P}$-algebra $A$, we define a map

$$
\operatorname{hom}_{\ell}^{\alpha}(y, 1): \mathrm{B}_{\iota} \operatorname{hom}^{\alpha}(C, A) \longrightarrow \operatorname{hom}^{\alpha}\left(C^{\prime}, A\right)
$$

by sending $\mu_{n}^{\vee} \otimes F$ to the following map:

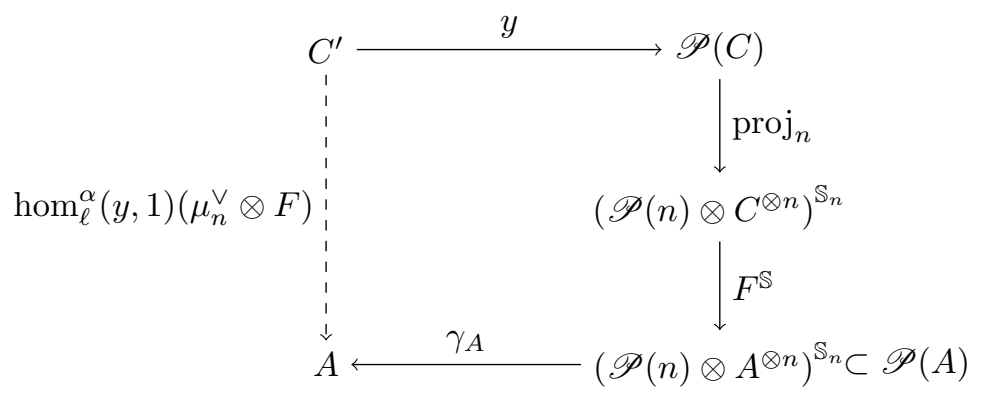

Here, we implicitly used the fact that we are working over a field of characteristic 0 to identify invariants and coinvariants.

The main result of this section is the following one.

Theorem 3.1. Let $\alpha: \mathscr{C} \rightarrow \mathscr{P}$ be a twisting morphism. Let $C^{\prime}, C$ be two $\mathscr{C}$-coalgebras, and let $A, A^{\prime}$ be two $\mathscr{P}$-algebras.

1. The map

$$
\operatorname{hom}_{r}^{\alpha}(1,-): \operatorname{hom}^{\alpha}\left(\mathrm{B}_{\alpha} A, A^{\prime}\right) \longrightarrow \operatorname{hom}^{\iota}\left(\mathrm{B}_{\iota} \operatorname{hom}^{\alpha}(C, A), \operatorname{hom}^{\alpha}\left(C, A^{\prime}\right)\right)
$$

is a strict morphism of $₫ \mathscr{L}_{\infty}$-algebras.

2. The map

$$
\operatorname{hom}_{\ell}^{\alpha}(-, 1): \operatorname{hom}^{\alpha}\left(C^{\prime}, \Omega_{\alpha} C\right) \longrightarrow \operatorname{hom}^{\iota}\left(\mathrm{B}_{\iota} \operatorname{hom}^{\alpha}(C, A), \operatorname{hom}^{\alpha}\left(C^{\prime}, A\right)\right)
$$

is a strict morphism of $\lrcorner \mathscr{L}_{\infty}$-algebras.

The proof of this result is technical, and we postpone it to Section 4.2.

\subsection{Infinity-morphisms and two bifunctors}

We will now begin to unravel the consequences of Theorem 3.1.

The reader might have recognized the diagrams defining the morphisms hom ${ }_{r}^{\alpha}(1,-)$ and $\operatorname{hom}_{\ell}^{\alpha}(-, 1)$, as they are very similar to the ones found in [RNW17, Sect. 5], which define two extensions of the functor $\operatorname{hom}^{\alpha}(-,-)$ to take $\infty_{\alpha}$-morphisms in one slot or the other. We can use Theorem 2.4 to easily recover one of the main results found in loc. cit. From now on we will identify $\infty_{\alpha}$-morphisms $\Psi: A \rightsquigarrow A^{\prime}$ of $\mathscr{P}$-algebras with the associated Maurer-Cartan elements $\Psi \in \operatorname{hom}^{\alpha}\left(\mathrm{B}_{\alpha} A, A^{\prime}\right)$, and similarly for $\infty_{\alpha}$-morphisms of $\mathscr{C}$-coalgebras.

Corollary 3.2 ([RNW17, Thm. 5.1]). Let $\alpha: \mathscr{C} \rightarrow \mathscr{P}$ be a twisting morphism. Let $C^{\prime}, C$ be two $\mathscr{C}$-coalgebras, and let $A, A^{\prime}$ be two $\mathscr{P}$-algebras.

1. Let $\Psi: A \rightsquigarrow A^{\prime}$ be an $\infty_{\alpha}$-morphism of $\mathscr{P}$-algebras. Then

$$
\operatorname{hom}_{r}^{\alpha}(1, \Psi): \operatorname{hom}^{\alpha}(C, A) \rightsquigarrow \operatorname{hom}^{\alpha}\left(C, A^{\prime}\right)
$$

is an $\infty$-morphism of $\lrcorner \mathscr{L}_{\infty}$-algebras. 
2. Let $\Phi: C^{\prime} \rightsquigarrow C$ be an $\infty_{\alpha}$-morphism of $\mathscr{C}$-coalgebras. Then

$$
\operatorname{hom}_{\ell}^{\alpha}(\Phi, 1): \operatorname{hom}^{\alpha}(C, A) \rightsquigarrow \operatorname{hom}^{\alpha}\left(C^{\prime}, A\right)
$$

is an $\infty$-morphism of $₫ \mathscr{L}_{\infty}$-algebras.

Proof. We prove only the first statement, the proof of the second one being completely analogous. The $\infty_{\alpha}$-morphism $\Psi$ corresponds to a Maurer-Cartan element in $\operatorname{hom}^{\alpha}\left(\mathrm{B}_{\alpha} A, A^{\prime}\right)$, which we denote again by $\Psi$ by abuse of notation. Since the map $\operatorname{hom}_{r}^{\alpha}(1,-)$ is a morphism of $\triangleleft \mathscr{L}_{\infty}$-algebras by Theorem 3.1, it preserves MaurerCartan elements, so that $\operatorname{hom}_{r}^{\alpha}(1, \Psi)$ is a Maurer-Cartan element of the $\triangleleft \mathscr{L}_{\infty}$-algebra $\operatorname{hom}^{\iota}\left(\mathrm{B}_{\iota} \operatorname{hom}^{\alpha}(C, A), \operatorname{hom}^{\alpha}\left(C, A^{\prime}\right)\right)$. But this is equivalent to say that $\operatorname{hom}_{r}^{\alpha}(1, \Psi)$ is an $\infty$-morphism of $s \mathscr{L}_{\infty}$-algebras from $\operatorname{hom}^{\alpha}(C, A)$ to $\operatorname{hom}^{\alpha}\left(C, A^{\prime}\right)$, as we wanted.

But Theorem 3.1 gives us even more than that.

Corollary 3.3. Let $\alpha: \mathscr{C} \rightarrow \mathscr{P}$ be a twisting morphism. Let $C^{\prime}, C$ be two $\mathscr{C}$-coalgebras, and let $A, A^{\prime}$ be two $\mathscr{P}$-algebras.

1. Let $\Psi, \Psi^{\prime}: A \rightsquigarrow A^{\prime}$ be two $\infty_{\alpha}$-morphisms of $\mathscr{P}$-algebras. If $\Psi$ and $\Psi^{\prime}$ are homotopic, then so are the two induced $\infty$-morphisms of $\triangleleft \mathscr{L}_{\infty}$-algebras $\operatorname{hom}_{r}^{\alpha}(1, \Psi)$ and $\operatorname{hom}_{r}^{\alpha}\left(1, \Psi^{\prime}\right)$.

2. Let $\Phi, \Phi^{\prime}: C^{\prime} \rightsquigarrow C$ be two $\infty_{\alpha}$-morphisms of $\mathscr{C}$-coalgebras. If $\Phi$ and $\Phi^{\prime}$ are homotopic, then so are the $\infty$-morphisms of $\lrcorner \mathscr{L}_{\infty}$-algebras $\operatorname{hom}_{\ell}^{\alpha}(\Phi, 1)$ and $\operatorname{hom}_{\ell}^{\alpha}\left(\Phi^{\prime}, 1\right)$.

Proof. Again, we will only prove the first statement. The fact that $\Psi$ and $\Psi^{\prime}$ are homotopic is equivalent to saying that the associated Maurer-Cartan elements of $\operatorname{hom}^{\alpha}\left(\mathrm{B}_{\alpha} A, A^{\prime}\right)$ are gauge equivalent (by Theorem 2.4). Since the map $\operatorname{hom}_{r}^{\alpha}(1,-)$ is a morphism of $s \mathscr{L}_{\infty}$-algebras, this implies that the Maurer-Cartan elements $\operatorname{hom}_{r}^{\alpha}(1, \Psi)$ and $\operatorname{hom}_{r}^{\alpha}\left(1, \Psi^{\prime}\right)$ are also gauge equivalent, which is equivalent to say that the associated $\infty$-morphisms of $₫ \mathscr{L}_{\infty}$-algebras are homotopic.

There is one important but straightforward fact that was not proven in [RNW17], which relates the morphisms $\operatorname{hom}_{r}^{\alpha}(1,-)$ and $\operatorname{hom}_{\ell}^{\alpha}(-, 1)$, and compositions of morphisms. Recall that the action of an $\infty$-morphisms $\Theta: \mathfrak{g} \rightarrow \mathfrak{h}$ of $\triangleleft \mathscr{L}_{\infty}$-algebras on Maurer-Cartan elements is given by

$$
\operatorname{MC}(\Theta)(x):=\sum_{n \geqslant 1} \frac{1}{n !} \theta_{n}(x, \ldots, x) \in \operatorname{MC}(\mathfrak{h})
$$

on $x \in \mathrm{MC}(\mathfrak{g})$.

Proposition 3.4. Let $\alpha: \mathscr{C} \rightarrow \mathscr{P}$ be a twisting morphism. Let $C^{\prime}, C$ be two $\mathscr{C}$-coalgebras, and let $A, A^{\prime}$ be two $\mathscr{P}$-algebras.

1. Let $\Psi: A \rightsquigarrow A^{\prime}$ be an $\infty_{\alpha}$-morphism of $\mathscr{P}$-algebras, and let $f: C \rightarrow \mathrm{B}_{\alpha} A$ be a morphism of $\mathscr{C}$-coalgebras, which we see as a Maurer-Cartan element of $\operatorname{hom}^{\alpha}(C, A)$. Then

$$
\operatorname{hom}_{r}^{\alpha}(1, \Psi)(f)=\left(C \stackrel{f}{\longrightarrow} \mathrm{B}_{\alpha} A \stackrel{\Psi}{\longrightarrow} \mathrm{B}_{\alpha} A^{\prime}\right)
$$

is a morphism of $\mathscr{C}$-coalgebras. 
2. Let $\Phi: C^{\prime} \rightsquigarrow C$ be an $\infty_{\alpha}$-morphism of $\mathscr{C}$-coalgebras, and let $g: \Omega_{\alpha} C \rightarrow A$ be a morphism of $\mathscr{P}$-algebras, which we see as a Maurer-Cartan element of $\operatorname{hom}^{\alpha}(C, A)$. Then

$$
\operatorname{hom}_{\ell}^{\alpha}(\Phi, 1)(g)=\left(\Omega_{\alpha} C^{\prime} \stackrel{\Phi}{\longrightarrow} \Omega_{\alpha} C \stackrel{g}{\longrightarrow} A\right)
$$

is a morphism of $\mathscr{P}$-algebras.

Proof. In order to give a clear proof, we will write $\tilde{f} \in \operatorname{MC}\left(\operatorname{hom}^{\alpha}(C, A)\right)$ for the element $f$ seen as a linear map $C \rightarrow A$, and $f$ for the equivalent map of $\mathscr{C}$-coalgebras $C \rightarrow \mathrm{B}_{\alpha} A$. We pass from the former to the latter by

$$
f=\left(1_{\mathscr{C}} \circ \widetilde{f}\right) \Delta_{C} .
$$

When writing $\Psi$, we will mean the map of $\mathscr{C}$-coalgebras $\mathrm{B}_{\alpha} A \rightarrow \mathrm{B}_{\alpha} A^{\prime}$, and the associated Maurer-Cartan element is

$$
\widetilde{\Psi}:=\operatorname{proj}_{A^{\prime}} \Psi \in \operatorname{MC}\left(\operatorname{hom}^{\alpha}\left(\mathrm{B}_{\alpha} A, A^{\prime}\right)\right) .
$$

With this notation, we have

$$
\begin{aligned}
\operatorname{MC}_{\left(\operatorname{hom}_{r}^{\alpha}(1, \widetilde{\Psi})\right)(\widetilde{f})} & =\sum_{n \geqslant 1} \frac{1}{n !} \operatorname{hom}_{r}^{\alpha}(1, \widetilde{\Psi})\left(\mu_{n}^{\vee} \otimes \widetilde{f}^{\otimes n}\right) \\
& =\sum_{n \geqslant 1} \frac{1}{n !} \widetilde{\Psi}\left(\widetilde{f}^{\otimes n}\right)^{\mathbb{S}} \Delta_{C}^{n} \\
& =\sum_{n \geqslant 1} \operatorname{proj}_{A^{\prime}} \Psi\left(1_{\mathscr{C}} \circ \widetilde{f}\right) \Delta_{C}^{n} \\
& =\operatorname{proj}_{A^{\prime}} \Psi\left(1_{\mathscr{C}} \circ \widetilde{f}\right) \Delta_{C} \\
& =\operatorname{proj}_{A^{\prime}} \Psi f,
\end{aligned}
$$

where $\mu_{1}^{\vee}=$ id. Here, $\operatorname{MC}\left(\operatorname{hom}_{r}^{\alpha}(1, \widetilde{\Psi})\right)$ denotes the map induced on the set of MaurerCartan elements of $\operatorname{hom}^{\alpha}(C, A)$ by the morphism of $s \mathscr{L}_{\infty}$-algebras $\operatorname{hom}_{r}^{\alpha}(1, \widetilde{\Psi})$. The other case is similar, and left to the reader.

In particular, we can take $C=\mathrm{B}_{\alpha} A^{\prime \prime}$ in Proposition 3.4, so that $f$ is an $\infty_{\alpha^{-}}$ morphism of $\mathscr{P}$-algebras $A^{\prime \prime} \rightsquigarrow A$, and we recover compositions of $\infty_{\alpha}$-morphisms. In particular, Proposition 3.4 immediately implies that

$$
\operatorname{hom}_{r}^{\alpha}(1, \Psi) \operatorname{hom}_{r}^{\alpha}\left(1, \Psi^{\prime}\right)=\operatorname{hom}_{r}^{\alpha}\left(1, \Psi \Psi^{\prime}\right)
$$

for composable $\infty_{\alpha}$-morphisms of $\mathscr{P}$-algebras, and that

$$
\operatorname{hom}_{\ell}^{\alpha}\left(\Phi^{\prime}, 1\right) \operatorname{hom}_{\ell}^{\alpha}(\Phi, 1)=\operatorname{hom}_{\ell}^{\alpha}\left(\Phi \Phi^{\prime}, 1\right)
$$

for composable $\infty_{\alpha}$-morphisms of $\mathscr{C}$-coalgebras (notice the contravariance). Thus, we recover another important result. 
Corollary 3.5 ([RNW17, Cor. 5.4]). The bifunctor

$$
\left.\operatorname{hom}^{\alpha}:(\mathscr{C} \text {-cog })^{\text {op }} \times \mathscr{P} \text {-alg } \longrightarrow\right\lrcorner \mathscr{L}_{\infty} \text {-alg }
$$

extends to two bifunctors

$$
\operatorname{hom}_{r}^{\alpha}:(\mathscr{C} \text {-cog })^{\mathrm{op}} \times \infty_{\alpha}-\mathscr{P} \text {-alg } \longrightarrow \triangleleft \mathscr{L}_{\infty} \text {-alg }
$$

and

$$
\left.\operatorname{hom}_{\ell}^{\alpha}:\left(\infty_{\alpha}-\mathscr{C}-\operatorname{cog}\right)^{\text {op }} \times \mathscr{P} \text {-alg } \longrightarrow\right\lrcorner \mathscr{L}_{\infty} \text {-alg } .
$$

Proof. It is straightforward to check that if $f: C^{\prime} \rightarrow C$ is a strict morphism of $\mathscr{C}$ coalgebras, and $\Psi$ is an $\infty_{\alpha}$-morphism of $\mathscr{P}$-algebras, then

$$
\operatorname{hom}^{\alpha}(f, 1) \operatorname{hom}_{r}^{\alpha}(1, \Psi)=\operatorname{hom}_{r}^{\alpha}(1, \Psi) \operatorname{hom}^{\alpha}(f, 1) .
$$

What said above then concludes the proof that $\operatorname{hom}_{r}^{\alpha}(-,-)$ is a bifunctor. The proof for $\operatorname{hom}_{\ell}^{\alpha}(-,-)$ is analogous.

\subsection{The two bifunctors commute up to homotopy}

Having extended the bifunctor hom ${ }^{\alpha}(-,-)$ to the two bifunctors $\operatorname{hom}_{r}^{\alpha}(-,-)$ and $\operatorname{hom}_{\ell}^{\alpha}(-,-)$ accepting $\infty_{\alpha}$-morphisms in the right and left slot respectively, it is natural to ask if those two functors admit a common extension to a bifunctor accepting $\infty_{\alpha}$-morphisms in both slots simultaneously. Unfortunately, this is not possible, as was proven by the authors in [RNW17, Sect. 6]. In the present paper, we will prove the next best thing.

Theorem 3.6. Let $\alpha: \mathscr{C} \rightarrow \mathscr{P}$ be a Koszul twisting morphism. Let $C^{\prime}, C$ be two $\mathscr{C}-$ coalgebras and $\Phi: C^{\prime} \rightsquigarrow C$ an $\infty_{\alpha}$-morphism between them, and let $A, A^{\prime}$ be two $\mathscr{P}$ algebras and $\Psi: A \rightsquigarrow A^{\prime}$ an $\infty_{\alpha}$-morphism between them. The two composites

$$
\operatorname{hom}_{\ell}^{\alpha}(\Phi, 1) \operatorname{hom}_{r}^{\alpha}(1, \Psi) \quad \text { and } \operatorname{hom}_{r}^{\alpha}(1, \Psi) \operatorname{hom}_{\ell}^{\alpha}(\Phi, 1)
$$

are homotopic as $\infty$-morphisms of $s \mathscr{L}_{\infty}$-algebras from $\operatorname{hom}^{\alpha}(C, A)$ to $\mathrm{hom}^{\alpha}\left(C^{\prime}, A^{\prime}\right)$.

The proof of this result is postponed to Section 4.3.

Remark 3.7. The assumption that $\alpha$ is Koszul in this result cannot be removed although we do not exclude that it might be weakened. Counterexamples to the conclusion of Theorem 3.6 for $\alpha$ not Koszul can easily be constructed, e.g., by taking $\alpha$ to be the zero twisting morphism. We will give such a counterexample in Appendix A.

An immediate corollary is the following result.

Corollary 3.8. Let $\alpha: \mathscr{C} \rightarrow \mathscr{P}$ be a Koszul twisting morphism. The bifunctor

$$
\operatorname{hom}^{\alpha}:(\mathscr{C} \text {-cog })^{\text {op }} \times \mathscr{P} \text {-alg } \longrightarrow \triangleleft \mathscr{L}_{\infty} \text {-alg }
$$

extends to a bifunctor

$$
\left.\operatorname{Ho}\left(\operatorname{hom}^{\alpha}\right): \operatorname{Ho}\left(\infty_{\alpha}-\mathscr{C} \text {-cog }\right)^{\text {op }} \times \operatorname{Ho}\left(\infty_{\alpha}-\mathscr{P} \text {-alg }\right) \longrightarrow \operatorname{Ho}(\infty-\lrcorner \mathscr{L}_{\infty} \text {-alg }\right)
$$

between the respective homotopy categories which restricts to $\operatorname{hom}_{\ell}^{\alpha}$ and $\operatorname{hom}_{r}^{\alpha}$ on the evident subcategories. 


\section{Proof of Theorems 2.4, 3.1, and 3.6}

This section collects all of the proofs we have postponed in the rest of the article in order to improve readability.

\subsection{Proof of Theorem 2.4}

We will prove the following theorem, which we find of independent interest. To prove Theorem 2.4 we will only need the case of the 0th homotopy group, but proving the whole result does not require a much bigger amount of work. We denote by $\Omega$. the simplicial commutative algebra given by the polynomial de Rham forms on the simplices, and for an $\lrcorner \mathscr{L}_{\infty}$-algebra $\mathfrak{g}$ we denote by

$$
\operatorname{MC} \bullet(\mathfrak{g}):=\operatorname{MC}\left(\mathfrak{g} \otimes \Omega_{\bullet}\right)
$$

the simplicial set of Maurer-Cartan elements. Notice that this definition does not make sense if one does not assume some kind of "finiteness" condition on $\mathfrak{g}$, since the Maurer-Cartan equation is an infinite sum of elements in a chain complex. The appropriate condition in this context is requiring that $\mathfrak{g}$ carries a sensible filtration with respect to which it is complete, see e.g., [RNW17, Def. 8.1]. Since in the present paper we work exclusively with conilpotent coalgebras, all the convolution algebras appearing automatically satisfy this condition, as we will see shortly.

Theorem 4.1. Let $\alpha: \mathscr{C} \rightarrow \mathscr{P}$ be a twisting morphism, let $C$ be a $\mathscr{C}$-coalgebra, and let $A$ be a $\mathscr{P}$-algebra. Then we have a natural homotopy equivalence of simplicial sets

$$
\mathrm{MC} \bullet\left(\operatorname{hom}^{\alpha}(C, A)\right) \simeq \mathrm{MC}\left(\operatorname{hom}^{\alpha}\left(C, A \otimes \Omega_{\bullet}\right)\right) .
$$

induced by the canonical inclusion

$$
\Theta: \operatorname{hom}(C, A) \otimes \Omega_{\bullet} \stackrel{\cong}{\longrightarrow} \operatorname{hom}\left(C, A \otimes \Omega_{\bullet}\right)
$$

given on pure tensors by sending $\phi \otimes \omega$ with $\phi \in \operatorname{hom}(C, A)$ and $\omega \in \Omega_{\bullet}$ to

$$
\Theta(\phi \otimes \omega)=(c \in C \longmapsto \phi(c) \otimes \omega) .
$$

Here, the $\lrcorner \mathscr{L}_{\infty}$-algebra $\operatorname{hom}^{\alpha}(C, A)$ is filtered by

$$
\mathcal{F}_{n} \operatorname{hom}^{\alpha}(C, A):=\left\{\phi \in \operatorname{hom}(C, A) \mid \mathcal{F}_{n}^{\text {corad }} C \subseteq \operatorname{ker} \phi\right\},
$$

where $\mathscr{F}_{\bullet}^{\text {corad }} C$ is the coradical filtration of $C$, see e.g., [LV12, Sect. 5.8.4], which is exhaustive since we supposed that all coalgebras are conilpotent. This makes $\operatorname{hom}^{\alpha}(C, A)$ into a complete $s \mathscr{L}_{\infty}$-algebra, so that it makes sense to define the MaurerCartan space $\mathrm{MC} \bullet\left(\operatorname{hom}^{\alpha}(C, A)\right)$. A similar filtration is put on $\operatorname{hom}^{\alpha}\left(C, A \otimes \Omega_{n}\right)$, for all $n \geqslant 0$. Complete $\lrcorner \mathscr{L}_{\infty}$-algebra were called filtered $s \mathscr{L}_{\infty}$-algebras in $[\mathbf{R N W} 17$, Def. 8.1].

One would like to go the easy way, and to prove the statement simply by saying that $\operatorname{hom}^{\alpha}\left(C, A \otimes \Omega_{\bullet}\right)$ is isomorphic to $\operatorname{hom}^{\alpha}(C, A) \otimes \Omega_{\bullet}$. However, since $\Omega_{\bullet}$ is infinite dimensional this is not true unless $C$ is finite dimensional. We can work around this problem as follows. There is a contraction due to Dupont [Dup76]

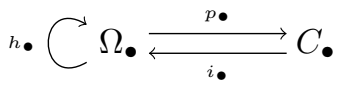


from $\Omega_{\bullet}$ to a simplicial sub-complex $C_{\bullet}$. This sub-complex can be thought of as the dual of the cellular complex of the geometric simplices. In particular, it is finite dimensional in every simplicial degree. The reader desiring more detail should consult the original article [Dup76], or e.g., [Get09, Sect. 3]. Then, we proceed as follows:

1. We transfer the simplicial $₫ \mathscr{L}_{\infty}$-algebra structure from $\operatorname{hom}^{\alpha}\left(C, A \otimes \Omega_{\bullet}\right)$ to $\operatorname{hom}\left(C, A \otimes C_{\bullet}\right)$ using the Dupont contraction, and prove that the simplicial sets obtained from these algebras by taking the Maurer-Cartan elements are homotopy equivalent.

2. We prove that the simplicial $\lrcorner \mathscr{L}_{\infty}$-algebra $\operatorname{hom}\left(C, A \otimes C_{\bullet}\right)$ thus obtained is isomorphic to the simplicial $₫ \mathscr{L}_{\infty}$-algebra $\operatorname{hom}(C, A) \otimes C$ • similarly obtained from $\operatorname{hom}^{\alpha}(C, A) \otimes \Omega$. by homotopy transfer theorem.

3. We conclude by using some results of [RN17] to prove that the simplicial sets of Maurer-Cartan elements of hom $(C, A) \otimes C_{\bullet}$ and of $\operatorname{hom}^{\alpha}(C, A) \otimes \Omega_{\bullet}$ are homotopy equivalent.

The proof of point (1) uses methods very similar to the ones used to prove [RN17, Thm. 3.3], whose demonstration is itself inspired from the proof of the DolgushevRogers theorem [DR15], and which implies point (3).

Now to make the details more precise. The Dupont contraction induces a contraction

$$
\left(1_{A} \otimes h_{\bullet}\right)_{*} \bigcirc \operatorname{hom}^{\alpha}\left(C, A \otimes \Omega_{\bullet}\right) \underset{\left(1_{A} \otimes i_{\bullet}\right)_{*}}{\stackrel{\left(1_{A} \otimes p_{\bullet}\right)_{*}}{\longleftarrow}} \operatorname{hom}\left(C, A \otimes C_{\bullet}\right)
$$

By the homotopy transfer theorem - e.g. [LV12, Sect. 10.3] — we can endow $\operatorname{hom}\left(C, A \otimes C_{\bullet}\right)$ with an $\triangleleft \mathscr{L}_{\infty}$-algebra structure and extend $\left(1_{A} \otimes p_{\bullet}\right)_{*}$ and $\left(1_{A} \otimes i_{\bullet}\right)_{*}$ to simplicial $\infty_{\iota}$-morphisms of $\lrcorner \mathscr{L}_{\infty}$-algebras, where as usual $\iota: \mathrm{Com}^{\vee} \rightarrow \triangleleft \mathscr{L}_{\infty}$ is the natural twisting morphism. We denote by

$$
P_{\bullet}: \operatorname{MC}\left(\operatorname{hom}^{\alpha}\left(C, A \otimes \Omega_{\bullet}\right)\right) \longrightarrow \mathrm{MC}_{\bullet}\left(\operatorname{hom}^{\alpha}(C, A)\right)
$$

and

$$
I_{\bullet}: \mathrm{MC}_{\bullet}\left(\operatorname{hom}^{\alpha}(C, A)\right) \longrightarrow \mathrm{MC}\left(\operatorname{hom}^{\alpha}\left(C, A \otimes \Omega_{\bullet}\right)\right)
$$

the morphisms of simplicial sets induced by these $\infty$-morphisms on the MaurerCartan sets. The following result implies point (1).

Proposition 4.2. The morphisms of simplicial sets $P_{\bullet}$ and $I_{\bullet}$ are homotopy inverses to each other.

Proof. The proof of [RN17, Thm. 3.3] goes through essentially unchanged by replacing $\mathfrak{g} \otimes \Omega_{\bullet}$ by $\operatorname{hom}^{\alpha}\left(C, A \otimes \Omega_{\bullet}\right)$, and $\mathfrak{g} \otimes C_{\bullet}$ by hom $\left(C, A \otimes C_{\bullet}\right)$. Therefore, we will only give a sketch of the proof here, and refer to op. cit. for the details.

First of all, the composite $P_{\bullet} I_{\bullet}$ is the identity, cf. [RN17, Lemma 3.5]. Therefore, it is enough to prove that the composite

$$
R_{\bullet}:=I_{\bullet} P_{\bullet}: \operatorname{MC}\left(\operatorname{hom}^{\alpha}\left(C, A \otimes \Omega_{\bullet}\right)\right) \longrightarrow \operatorname{MC}\left(\operatorname{hom}^{\alpha}\left(C, A \otimes \Omega_{\bullet}\right)\right)
$$

is a weak equivalence. This is done by an inductive procedure on the filtration, and then passing to the limit. 
The map $R_{0}$ is simply given by the identity, and thus induces a bijection at the level of the 0th homotopy group. Then one considers the case where $C=\mathscr{F}_{2}^{\text {corad }} C$, where $\operatorname{hom}^{\alpha}(C,-)$ lands in abelian $\triangleleft \mathscr{L}_{\infty}$-algebras, and proves that $R_{\bullet}$ is a weak equivalence of simplicial sets in that case, with the same methods as [RN17, Lemma 3.7]. Further, one notices that [RN17, Lemmas 3.8 and 3.10] also hold in this context, so that all of the arguments carry over to the present situation, giving us the result we wanted.

The other contraction we consider is

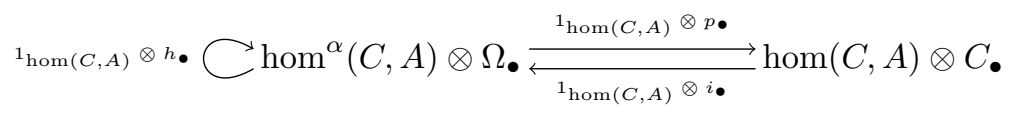

Again, the homotopy transfer theorem gives us an $\triangleleft \mathscr{L}_{\infty}$-algebra structure on the chain complex $\operatorname{hom}(C, A) \otimes C_{\bullet}$. Since $C_{\bullet}$ is finite dimensional in every simplicial degree, we have a natural isomorphism of simplicial chain complexes

$$
\widetilde{\Theta}: \operatorname{hom}(C, A) \otimes C_{\bullet} \stackrel{\cong}{\longrightarrow} \operatorname{hom}\left(C, A \otimes C_{\bullet}\right)
$$

given on pure tensors by sending $\phi \otimes \omega$ with $\phi \in \operatorname{hom}(C, A)$ and $\omega \in C$ • to

$$
\widetilde{\Theta}(\phi \otimes \omega)=(c \in C \longmapsto \phi(c) \otimes \omega) .
$$

We check that $\widetilde{\Theta}$ respects the transferred $\diamond \mathscr{L}_{\infty}$-structures.

Proposition 4.3. The map $\widetilde{\Theta}$ is an isomorphism of $\triangleleft \mathscr{L}_{\infty}$-algebras with respect to the two transferred structures.

Proof. We consider the diagram

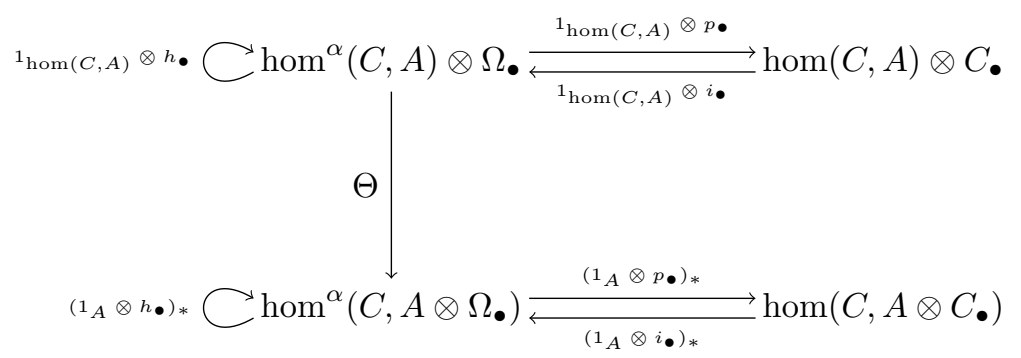

We have that $\Theta$ preserves the filtrations, and

$$
\Theta\left(1_{\text {hom }(C, A)} \otimes h_{\bullet}\right)=\left(1_{A} \otimes h_{\bullet}\right)_{*} \Theta .
$$

Therefore, the morphism $\Theta$ is a morphism of complete contractions in the sense of [Ban17, Def. 1.7]. Since $\Theta$ is a morphism of $s \mathscr{L}_{\infty}$-algebras, then by [Ban17, Lemma 1.10] we have that

$$
\operatorname{pr}_{2}(\Theta):=\left(1_{\text {hom }(C, A)} \otimes p_{\bullet}\right) \Theta\left(1_{A} \otimes i_{\bullet}\right)_{*}
$$

is a morphism of $\lrcorner \mathscr{L}_{\infty}$-algebras between the transfered $\lrcorner \mathscr{L}_{\infty}$-algebras. But 


$$
\begin{aligned}
\left(1_{\mathrm{hom}(C, A)} \otimes p_{\bullet}\right) \Theta\left(1_{A} \otimes i_{\bullet}\right)_{*}(\phi \otimes \omega) & =\left(1_{\mathrm{hom}(C, A)} \otimes p_{\bullet}\right) \Theta(\phi \otimes i(\omega)) \\
& =\left(1_{\mathrm{hom}(C, A)} \otimes p_{\bullet}\right)(c \mapsto \phi(c) \otimes i(\omega)) \\
& =(c \mapsto \phi(c) \otimes p i(\omega)) \\
& =(c \mapsto \phi(c) \otimes \omega) \\
& =\widetilde{\Theta}(\phi \otimes \omega)
\end{aligned}
$$

for any $\phi \in \operatorname{hom}(C, A)$ and $\omega \in C_{\bullet}$. This concludes the proof.

Now we can conclude the proof of the theorem.

Proof of Theorem 4.1. We have taken care of points (1) and (2) in our program. Point (3) is given by [RN17, Thm. 3.3]. Putting these steps together, we obtain the desired homotopy equivalence.

To prove that $\operatorname{MC}(\Theta)$ is indeed a homotopy equivalence, we refine slightly the proof of Proposition 4.3 and notice that the second part of [Ban17, Lemma 1.10], together with the fact that

$$
\left(1_{\mathrm{hom}(C, A)} \otimes p_{\bullet}\right)_{\infty}\left(1_{\mathrm{hom}(C, A)} \otimes i_{\bullet}\right)_{\infty}=1_{\mathrm{hom}(C, A) \otimes C \bullet},
$$

implies that

$$
P_{\bullet} \operatorname{MC}(\Theta) I_{\bullet}=\operatorname{MC}(\widetilde{\Theta}) .
$$

Since all the morphisms except - a priori - $\mathrm{MC}(\Theta)$ are homotopy equivalences, we conclude that $\mathrm{MC}(\Theta)$ must also be one, concluding the proof of the theorem.

As a consequence, we have the following.

Proof of Theorem 2.4. As mentioned in Remark 2.6, the proof of the fact that Maurer-Cartan elements corresponds to morphisms of algebras and coalgebras was done in [Wie16, Thm. 7.1(1)] and in a special case in [RN18a, Thm. 6.3].

We are left to prove points (1) and (2) of the statement. For this, notice that if $A$ is a $\mathscr{P}$-algebra, then $A \otimes \Omega_{1}$ is a good path object for $A$. This is seen by considering the two morphisms of $\mathscr{P}$-algebras

$$
A \longrightarrow A \otimes \Omega_{1} \longrightarrow A \times A,
$$

the first one sending $a \in A$ to $a \otimes 1 \in A \otimes \Omega_{1}$ and the second one sending $a\left(t_{0}, t_{1}\right) \in$ $A \otimes \Omega_{1}$ to $(a(1,0), a(0,1)) \in A \times A$. Therefore, two morphisms of $\mathscr{P}$-algebras

$$
f_{0}, f_{1}: \Omega_{\alpha} C \longrightarrow A
$$

are homotopic if and only if there exists a morphism of $\mathscr{P}$-algebras

$$
H: \Omega_{\alpha} C \longrightarrow A \otimes \Omega_{1}
$$

such that $H$ equals $f_{0}$, respectively $f_{1}$, if we evaluate its image at $(1,0)$, resp. $(0,1)$. But this is exactly saying that

$$
H \in \operatorname{hom}^{\alpha}\left(C, A \otimes \Omega_{1}\right)
$$

is a 1-simplex going from $f_{0} \in \operatorname{hom}^{\alpha}(C, A)$ to $f_{1} \in \operatorname{hom}^{\alpha}(C, A)$, i.e., that $f_{0}$ and $f_{1}$ represent the same element of $\pi_{0} \mathrm{MC}\left(\operatorname{hom}^{\alpha}(C, A)\right)$. Finally, by Theorem 4.1 this is equivalent to the fact that $f_{0}$ and $f_{1}$ are in the same path component of $\mathrm{MC} \bullet\left(\operatorname{hom}^{\alpha}(C, A)\right)$, 
i.e., that they are gauge equivalent. This concludes the proof of point (2).

The proof of point (1) is done analogously using the fact that $\mathrm{B}_{\alpha}\left(A \otimes \Omega_{1}\right)$ is a good path object for $\mathrm{B}_{\alpha} A$, which we show now. The bar functor $\mathrm{B}_{\alpha}$ is a right Quillen functor by [DCH16, Thm. 3.11(1)], or by [Val14, Thm. 2.1.3] in the case where $\alpha$ is Koszul. Thus, it preserves fibrations, and by Ken Brown's lemma - see e.g., [Hov99, Lemma 1.1.12] — it also sends weak equivalences between fibrant objects to weak equivalences. But all $\mathscr{P}$-algebras are fibrant, and $\mathrm{B}_{\alpha}$ also preserves limits being right adjoint, so it follows that $\mathrm{B}_{\alpha}$ preserves good path objects. Therefore, $\mathrm{B}_{\alpha}\left(A \otimes \Omega_{1}\right)$ is a good path object for $\mathrm{B}_{\alpha} A$, and one concludes the proof of point (1) proceeding in the same way as before.

\subsection{Proof of Theorem 3.1}

We begin by proving a technical result that we will need in the main proof.

Lemma 4.4. Let $\mathscr{C}$ be a cooperad, and let $C$ be a conilpotent $\mathscr{C}$-coalgebra. Then

$$
\left(\Delta_{(1)} \circ 1_{C}\right) \Delta_{C}^{n}=\sum_{\substack{n_{1}+n_{2}=n+1 \\ 1 \leqslant i \leqslant n_{1}}}\left(1_{\mathscr{C}} \circ\left(1_{C}^{\otimes(i-1)} \otimes \Delta_{C}^{n_{2}} \otimes 1_{C}^{\otimes(n-i)}\right)\right) \Delta_{C}^{n_{1}} .
$$

Moreover, let $f_{1}, \ldots, f_{n} \in \operatorname{hom}(C, V)$ for a chain complex $V$. Under the canonical inclusion

$$
\bigoplus_{\substack{n_{1}+n_{2}=n+1 \\ 1 \leqslant i \leqslant n_{1}}} \mathscr{C}\left(n_{1}\right) \circ\left(C^{\otimes(i-1)} \otimes\left(\mathscr{C}\left(n_{2}\right) \otimes C^{\otimes n_{2}}\right) \otimes C^{\otimes(n-i)}\right) \longleftrightarrow(\mathscr{C} \circ \mathscr{C})(n) \otimes C^{\otimes n}
$$

we have

$$
F^{\mathbb{S}}\left(\Delta_{(1)} \circ 1_{C}\right) \Delta_{C}^{n}=\sum_{S_{1} \sqcup S_{2}=[n]}(-1)^{\epsilon}\left(\left(F^{S_{1}} \Delta_{C}^{n_{1}}\right) \otimes F^{S_{2}}\right) \Delta_{C}^{n_{2}},
$$

where $n_{1}=\left|S_{1}\right|$ and $n_{2}=\left|S_{2}\right|+1$, and again $\epsilon$ is given by the Koszul sign rule because we are shuffling the maps $f_{i}$.

Proof. For the first identity, one considers the equality

$$
\left(\Delta_{\mathscr{C}} \circ 1_{C}\right) \Delta_{C}=\left(1_{\mathscr{C}} \circ \Delta_{C}\right) \Delta_{C}
$$

and then projects on the subspace

$$
(\mathscr{C} \circ(1) \mathscr{C})(C) \cong \mathscr{C} \circ(C ; \mathscr{C}(C)) .
$$

We leave the details to the reader. The second statement then follows in a straightforward way.

We can now prove the main result.

Proof of Theorem 3.1. We prove the first case, the second one being dual. We will begin by proving that the map $\operatorname{hom}_{r}^{\alpha}(1,-)$ commutes with the brackets, and then show that it also commutes with the differentials. All of this will be done by explicitly writing down and comparing the formulæ.

Let $x_{1}, \ldots, x_{k} \in \operatorname{hom}^{\alpha}\left(\mathrm{B}_{\alpha} A, A^{\prime}\right)$. Then we have

$$
\ell_{k}\left(x_{1}, \ldots, x_{k}\right)=\gamma_{A^{\prime}}(\alpha \otimes X)^{\mathbb{S}}\left(\Delta_{\mathscr{C}}^{k} \circ 1_{A}\right),
$$

where $X:=x_{1} \otimes \cdots \otimes x_{k}$, and thus for $f_{1}, \ldots, f_{n} \in \operatorname{hom}^{\alpha}(C, A)$ we get 


$$
\begin{aligned}
\operatorname{hom}_{r}^{\alpha}\left(1, \ell_{k}(X)\right)\left(\mu_{n}^{\vee} \otimes F\right)=\ell_{k}(X) F^{\mathbb{S}} \Delta_{C}^{n} \\
=\gamma_{A^{\prime}}(\alpha \otimes X)^{\mathbb{S}}\left(\Delta_{\mathscr{C}}^{k} \circ 1_{A}\right) F^{\mathbb{S}} \Delta_{C}^{n} \\
=\gamma_{A^{\prime}}(\alpha \otimes X)^{\mathbb{S}} F^{\mathbb{S}}\left(\Delta_{\mathscr{C}}^{k} \circ 1_{C}\right) \Delta_{C}^{n} \\
=\sum_{n_{1}+\cdots+n_{k}=n} \gamma_{A^{\prime}}(\alpha \otimes X)^{\mathbb{S}} F^{\mathbb{S}}\left(1_{\mathscr{C}} \circ\left(\Delta_{C}^{n_{1}} \otimes \cdots \otimes \Delta_{C}^{n_{k}}\right)\right) \Delta_{C}^{k} \\
=\sum_{S_{1} \sqcup \cdots \sqcup S_{k}=[n]}(-1)^{\epsilon_{1}} \gamma_{A^{\prime}}(\alpha \otimes X)^{\mathbb{S}}\left(1_{\mathscr{C}} \circ\left(F^{S_{1}} \Delta_{C}^{n_{1}} \otimes \cdots \otimes F^{S_{k}} \Delta_{C}^{n_{k}}\right)\right) \Delta_{C}^{k} \\
=\sum_{S_{1} \sqcup \cdots \sqcup S_{k}=[n]}(-1)^{\epsilon_{1}+\epsilon_{2}} \gamma_{A^{\prime}}\left(\alpha \circ 1_{A^{\prime}}\right)\left(1_{\mathscr{C}} \circ\left(x_{\sigma(1)} F^{S_{1}} \Delta_{C}^{n_{1}} \otimes \cdots \otimes x_{\sigma(k)} F^{S_{k}} \Delta_{C}^{n_{k}}\right)\right) \Delta_{C}^{k}
\end{aligned}
$$

where the fourth line follows from $\left(\Delta_{\mathscr{C}} \circ C\right) \Delta_{C}=\left(1_{\mathscr{C}} \circ \Delta_{C}\right) \Delta_{C}$, and in the passage from the fourth line to the fifth line we denoted $n_{i}:=\left|S_{i}\right|$. The Koszul signs are

$$
\epsilon_{1}=\sum_{i=1}^{k} \sum_{s \in S_{i}}\left|f_{s}\right| \sum_{j<i} \sum_{\substack{p \in S_{j} \\ p>s}}\left|f_{p}\right|
$$

obtained by shuffling the maps $f_{i}$, and $\epsilon_{2}$, which is similarly obtained by permuting the maps $x_{i}$ and interchanging them with the maps $f_{j}$.

On the other hand, we have

$$
\begin{aligned}
& \ell_{k}\left(\operatorname{hom}^{\alpha}(1, X)\right)\left(\mu_{n}^{\vee} \otimes F\right)=\left(\gamma_{\mathrm{hom}^{\alpha}\left(C, A^{\prime}\right)}\left(\iota \otimes \operatorname{hom}^{\alpha}(1, X)\right)^{\mathbb{S}}\left(\Delta_{\mathrm{Com}^{\vee}}^{k} \otimes 1_{\mathrm{hom}(C, A)}\right)\right)\left(\mu_{n}^{\vee} \otimes F\right) \\
& =\left(\gamma_{\operatorname{hom}^{\alpha}\left(C, A^{\prime}\right)}\left(\iota \otimes \operatorname{hom}^{\alpha}(1, X)\right)^{\mathbb{S}}\right)\left(\sum_{S_{1} \sqcup \ldots \sqcup S_{k}=[n]}(-1)^{\epsilon_{1}} \mu_{k}^{\vee} \otimes \bigotimes_{i=1}^{k}\left(\mu_{n_{i}}^{\vee} \otimes F^{S_{i}}\right)\right) \\
& =\gamma_{\mathrm{hom}^{\alpha}\left(C, A^{\prime}\right)}\left(\sum_{\substack{S_{1} \sqcup \ldots . S_{k}=[n] \\
\sigma \in \mathbb{S}_{k}}}(-1)^{\epsilon_{1}+\epsilon_{2}} s^{-1} \mu_{k}^{\vee} \otimes \bigotimes_{i=1}^{k} \operatorname{hom}^{\alpha}\left(1, x_{\sigma(i)}\right)\left(\mu_{n_{i}}^{\vee} \otimes F^{S_{i}}\right)\right) \\
& =\sum_{\substack{S_{1} \sqcup \ldots . S_{k \in S_{k}}=[n] \\
\sigma \in \mathbb{S}_{k}}}(-1)^{\epsilon_{1}+\epsilon_{2}} \gamma_{A^{\prime}}\left(\alpha \otimes \bigotimes_{i=1}^{k} \operatorname{hom}^{\alpha}\left(1, x_{\sigma(i)}\right)\left(\mu_{n_{i}}^{\vee} \otimes F^{S_{i}}\right)\right) \Delta_{C}^{k} \\
& =\sum_{\substack{S_{1} \sqcup \ldots \sqcup S_{k}=[n] \\
\sigma \in \mathbb{S}_{k}}}(-1)^{\epsilon_{1}+\epsilon_{2}} \gamma_{A^{\prime}}\left(\alpha \otimes \bigotimes_{i=1}^{k} x_{\sigma(i)} F^{S_{i}} \Delta_{C}^{n_{i}}\right) \Delta_{C}^{k} .
\end{aligned}
$$

Notice that in the fourth line we do not need to sum over permutations when applying the structure map $\gamma_{\mathrm{hom}^{\alpha}\left(C, A^{\prime}\right)}$, because the term

$$
\sum_{\substack{S_{1} \sqcup . . S_{k \in S_{k}}=[n] \\ \sigma \in \mathbb{S}_{k}}}(-1)^{\epsilon_{1}+\epsilon_{2}} s^{-1} \mu_{k}^{\vee} \otimes \bigotimes_{i=1}^{k} \operatorname{hom}^{\alpha}\left(1, x_{\sigma(i)}\right)\left(\mu_{n_{i}}^{\vee} \otimes F^{S_{i}}\right)
$$

in the third line naturally lives in invariants, not coinvariants.

In conclusion, we have

$$
\operatorname{hom}^{\alpha}\left(1, \ell_{k}(X)\right)=\ell_{k}\left(\operatorname{hom}^{\alpha}(1, X)\right) .
$$


We are left to prove that the morphism commutes with the differentials. In order to avoid cumbersome notation, we will denote simply by $d$ the differentials of both the $\lrcorner \mathscr{L}_{\infty}$-algebras $\operatorname{hom}^{\alpha}\left(\mathrm{B}_{\alpha} A, A^{\prime}\right)$ and $\operatorname{hom}^{\iota}\left(\mathrm{B}_{\iota} \operatorname{hom}^{\alpha}(C, A), \operatorname{hom}^{\alpha}\left(C, A^{\prime}\right)\right)$. The letter $\partial$ denotes instead the differential of $\operatorname{hom}(C, A)$. Let $x \in \operatorname{hom}^{\alpha}\left(\mathrm{B}_{\alpha} A, A^{\prime}\right)$, and let $f_{1}, \ldots, f_{n} \in \operatorname{hom}^{\alpha}(C, A)$. On one hand, we have

$$
\begin{aligned}
& d(x)=d_{A^{\prime}} x-(-1)^{|x|} x d_{\mathrm{B}_{\alpha} A} \\
&=d_{A^{\prime}} x-(-1)^{|x|} x d_{\mathscr{C} \circ A}-(-1)^{|x|} x\left(1_{\mathscr{C}} \circ\left(1_{A} ; \gamma_{A}\right)\right)\left(\left(1_{\mathscr{C}} \circ(1)\right.\right. \\
&\left.\alpha) \circ 1_{A}\right)\left(\Delta_{(1)} \circ 1_{A}\right),
\end{aligned}
$$

and thus

$$
\begin{aligned}
\operatorname{hom}^{\alpha}(1, d(x))( & \left.\mu_{n}^{\vee} \otimes F\right)=d(x) F^{\mathbb{S}} \Delta_{C}^{n} \\
= & d_{A^{\prime}} x F^{\mathbb{S}} \Delta_{C}^{n}-(-1)^{|x|} x d_{\mathscr{C} \circ A} F^{\mathbb{S}} \Delta_{C}^{n} \\
& -(-1)^{|x|} x\left(1_{\mathscr{C}} \circ\left(1_{A} ; \gamma_{A}\right)\right)\left(\left(1_{\mathscr{C}} \circ_{(1)} \alpha\right) \circ 1_{A}\right)\left(\Delta_{(1)} \circ 1_{A}\right) F^{\mathbb{S}} \Delta_{C}^{n} .
\end{aligned}
$$

The second term in (L1) equals

$$
\begin{aligned}
x d_{\mathscr{C} \circ A} F^{\mathbb{S}} \Delta_{C}^{n} & =x\left(d_{\mathscr{C}} \circ 1_{A}\right) F^{\mathbb{S}} \Delta_{C}^{n}+x\left(1_{\mathscr{C}} \circ^{\prime} d_{A}\right) F \Delta_{C}^{n} \\
& =(-1)^{|F|} x F^{\mathbb{S}}\left(d_{\mathscr{C}} \circ 1_{C}\right) \Delta_{C}^{n}+x \partial(F)^{\mathbb{S}} \Delta_{C}^{n}+(-1)^{|F|} x F\left(1_{\mathscr{C}} \circ^{\prime} d_{C}\right) \Delta_{C}^{n} \\
& =(-1)^{|F|} x F^{\mathbb{S}} d_{\mathscr{C}(C)} \Delta_{C}^{n}+x \partial(F)^{\mathbb{S}} \Delta_{C}^{n} \\
& =(-1)^{|F|} x F^{\mathbb{S}} \Delta_{C}^{n} d_{C}+x \partial(F)^{\mathbb{S}} \Delta_{C}^{n},
\end{aligned}
$$

while the term of (L2) gives

$$
\begin{aligned}
x & \left(1_{\mathscr{C}} \circ\left(1_{A} ; \gamma_{A}\right)\right)\left(\left(1_{\mathscr{C}} \circ_{(1)} \alpha\right) \circ 1_{A}\right)\left(\Delta_{(1)} \circ 1_{A}\right) F^{\mathbb{S}} \Delta_{C}^{n}= \\
= & x\left(1_{\mathscr{C}} \circ\left(1_{A} ; \gamma_{A}\right)\right)\left(\left(1_{\mathscr{C}} \circ(1) \alpha\right) \circ 1_{A}\right) F^{\mathbb{S}}\left(\Delta_{(1)} \circ 1_{C}\right) \Delta_{C}^{n} \\
= & \sum_{S_{1} \sqcup S_{2}=[n]}(-1)^{\epsilon} x\left(1_{\mathscr{C}} \circ\left(1_{A} ; \gamma_{A}\right)\right)\left(\left(1_{\mathscr{C}} \circ_{(1)} \alpha\right) \circ 1_{A}\right)\left(\left(F^{S_{1}} \Delta_{C}^{n_{1}}\right) \otimes F^{S_{2}}\right) \Delta_{C}^{n_{2}},
\end{aligned}
$$

where in the third line we used Lemma 4.4. On the other hand,

$$
d\left(\operatorname{hom}^{\alpha}(1, x)\right)=d_{\operatorname{hom}^{\alpha}\left(C, A^{\prime}\right)} \operatorname{hom}^{\alpha}(1, x)-(-1)^{\left|\operatorname{hom}^{\alpha}(1, x)\right|} \operatorname{hom}^{\alpha}(1, x) d_{\mathrm{B}_{\iota} \operatorname{hom}^{\alpha}(C, A)} .
$$

Notice that $(-1)^{\left|\operatorname{hom}^{\alpha}(1, x)\right|}=(-1)^{|x|}$. We apply this to $\mu_{n}^{\vee} \otimes F$ and obtain

$$
\begin{aligned}
d\left(\operatorname{hom}^{\alpha}(1, x)\right)\left(\mu_{n}^{\vee} \otimes F\right)= & d_{A^{\prime}} \operatorname{hom}^{\alpha}(1, x)-(-1)^{|x|+|F|} \operatorname{hom}^{\alpha}(1, x)\left(\mu_{n}^{\vee} \otimes F\right) d_{C} \\
& -(-1)^{|x|} \operatorname{hom}^{\alpha}(1, x)\left(d_{\mathrm{B}_{\iota} \operatorname{hom}^{\alpha}(C, A)}\left(\mu_{n}^{\vee} \otimes F\right)\right) .
\end{aligned}
$$

The first term equals $d_{A^{\prime}} x F \Delta_{C}^{n}$ and cancels with the first term of (L1), and the second term equals the first term of (T1). For the third term, we have

$$
\begin{aligned}
\operatorname{hom}^{\alpha}(1, x)\left(d_{\mathrm{B}_{\iota} \operatorname{hom}^{\alpha}(C, A)}\right. & \left.\left.\mu_{n}^{\vee} \otimes F\right)\right) \\
= & \operatorname{hom}^{\alpha}(1, x)\left(\mu_{n}^{\vee} \otimes \partial(F)\right) \\
& +\operatorname{hom}^{\alpha}(1, x)\left(\sum_{S_{1} \sqcup S_{2}=[n]} \mu_{n_{2}}^{\vee} \otimes\left(\ell_{n_{1}}\left(F^{S_{1}}\right) \otimes F^{S_{2}}\right)\right) .
\end{aligned}
$$

The first term of this expression cancels the second term of (T1). Therefore, we are left to show that (T3) equals (T2). We have 


$$
\begin{aligned}
\operatorname{hom}^{\alpha}(1, x) & \left(\sum_{S_{1} \sqcup S_{2}=[n]}(-1)^{\epsilon} \mu_{n_{2}}^{\vee} \otimes\left(\ell_{n_{1}}\left(F^{S_{1}}\right) \otimes F^{S_{2}}\right)\right) \\
= & \sum_{S_{1} \sqcup S_{2}=[n]}(-1)^{\epsilon} x\left(\ell_{n_{1}}\left(F^{S_{1}}\right) \otimes F^{S_{2}}\right) \Delta_{C}^{n_{2}} \\
= & \sum_{S_{1} \sqcup S_{2}=[n]}(-1)^{\epsilon} x\left(\left(\gamma_{A}\left(\alpha \otimes F^{S_{1}}\right) \Delta_{C}^{n_{1}}\right) \otimes F^{S_{2}}\right) \Delta_{C}^{n_{2}} \\
= & \sum_{S_{1} \sqcup S_{2}=[n]}(-1)^{\epsilon} x\left(1_{\mathscr{C}} \circ\left(\gamma_{A}\left(\alpha \circ 1_{A}\right) \otimes 1_{A}^{\otimes\left(n_{2}-1\right)}\right)\right)\left(1_{\mathscr{C}} \circ\left(F^{S_{1}} \Delta_{C}^{n_{1}} \otimes F^{S_{2}}\right)\right) \Delta_{C}^{n_{2}} \\
= & (\mathrm{T} 2)
\end{aligned}
$$

where $n_{1}=\left|S_{1}\right|$, and $n_{2}=\left|S_{2}\right|+1$. This concludes the proof.

\subsection{Proof of Theorem 3.6}

The strategy to prove that the two compositions are homotopic as $\infty$-morphisms of $\lrcorner \mathscr{L}_{\infty}$-algebras, or equivalently gauge equivalent as Maurer-Cartan elements, is as follows. We know that if $\Psi$ were a strict morphism, then the two compositions would commute. Therefore, we will rectify $\Psi$ to get a strict morphism $R(\Psi)$ of $\mathscr{P}$-algebras, which we do by applying the bar-cobar adjunction. However, to do this we need also to rectify the $\mathscr{P}$-algebras $A$ and $A^{\prime}$. Fortunately, if $\alpha$ is Koszul, then the new $\mathscr{P}_{-}$ algebras thus obtained are quasi-isomorphic to the original ones, and thus we are able to apply the Dolgushev-Rogers theorem [DR15, Thm. 1.1] to go back to the original two compositions and conclude the proof.

Proof of Theorem 3.6. Denote by $R(A):=\Omega_{\alpha} \mathrm{B}_{\alpha} A$ the bar-cobar resolution of $A$, and similarly for $A^{\prime}$. Since $\alpha$ is Koszul, the counit of the adjunction

$$
\epsilon_{A}: R(A) \longrightarrow A
$$

is a quasi-isomorphism by [LV12, Thm. 11.3.3]. The rectification of the $\infty_{\alpha}$-morphism $\Psi$ is given by the strict morphism

$$
R(\Psi): \Omega_{\alpha} \mathrm{B}_{\alpha} A \stackrel{\Omega_{\alpha} \Psi}{\longrightarrow} \Omega_{\alpha} \mathrm{B}_{\alpha} A^{\prime} .
$$

The proof is outlined by the diagram in Figure 1. The innermost square is commutative since $R(\Psi)$ is a strict morphism of $\mathscr{P}$-algebras, and the maps passing from the outer rim to the inner one are filtered quasi-isomorphisms. Notice that all squares are commutative, except for the outer one, which fails to be commutative at $\operatorname{hom}^{\alpha}(C, A)$.

Now consider the morphism of $\lrcorner \mathscr{L}_{\infty}$-algebras

$$
\begin{gathered}
\operatorname{hom}^{\iota}\left(\mathrm{B}_{\iota} \operatorname{hom}^{\alpha}(C, A), \operatorname{hom}^{\alpha}\left(C^{\prime}, A^{\prime}\right)\right) \\
\qquad \operatorname{hom}^{\iota}\left(\mathrm{B}_{\iota} \operatorname{hom}^{\alpha}\left(1, \epsilon_{A}\right), 1\right) \\
\operatorname{hom}^{\iota}\left(\mathrm{B}_{\iota} \operatorname{hom}^{\alpha}(C, R(A)), \operatorname{hom}^{\alpha}\left(C^{\prime}, A^{\prime}\right)\right)
\end{gathered}
$$

It is a filtered quasi-isomorphism, and it is given on Maurer-Cartan elements by precomposition with hom ${ }^{\alpha}\left(1, \epsilon_{A}\right)$. The two compositions

$$
\operatorname{hom}^{\alpha}(\Phi, 1) \operatorname{hom}^{\alpha}(1, \Psi) \text { and } \operatorname{hom}^{\alpha}(1, \Psi) \operatorname{hom}^{\alpha}(\Phi, 1)
$$




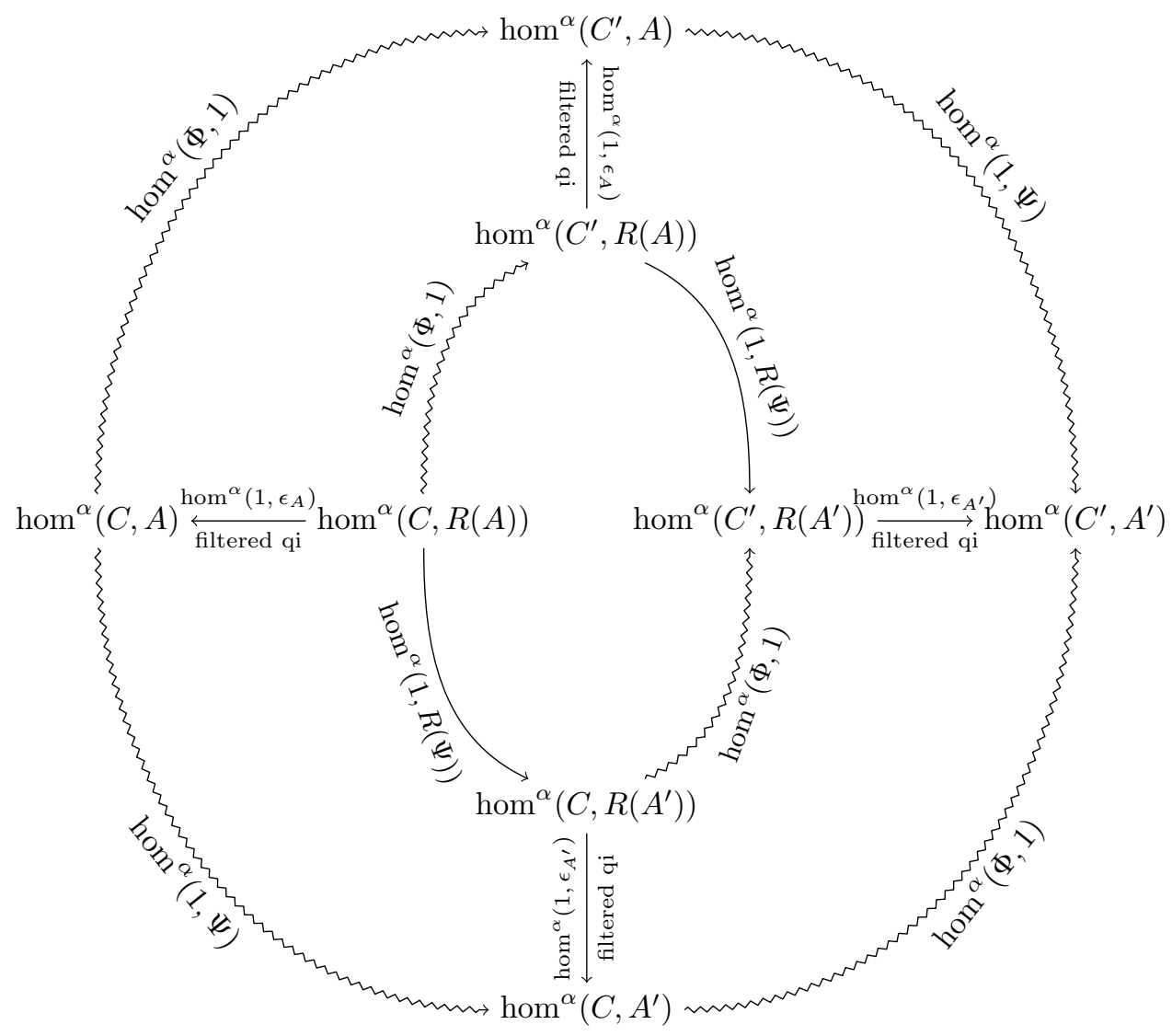

Figure 1: Outline of the proof of Theorem 3.6.

are naturally elements of $\operatorname{hom}^{\iota}\left(\mathrm{B}_{\iota} \operatorname{hom}^{\alpha}(C, A), \operatorname{hom}^{\alpha}\left(C^{\prime}, A^{\prime}\right)\right)$ and are mapped to the same elements, and thus, by the Dolgushev-Rogers theorem, they are homotopic.

Remark 4.5. The proof above supposes that we are filtering our convolution algebras with the filtration induced by a filtration on the $\mathscr{C}$-coalgebras - usually the coradical filtration. If one filters them by a filtration induced by filtrations on the $\mathscr{P}$-algebras, then the exact same proof goes through with the sole difference that one has to rectify the $\infty_{\alpha}$-morphism $\Phi$ instead of $\Psi$.

\section{Appendix A. A counterexample}

The goal of this appendix is to give an explicit counterexample to the conclusion of Theorem 3.6 in the case when the twisting morphism $\alpha$ is not Koszul.

More precisely, we will show that there exists a (non-Koszul) twisting morphism $\alpha: \mathscr{C} \rightarrow \mathscr{P}$, two $\mathscr{C}$-coalgebras $C^{\prime}, C$, an $\infty_{\alpha}$-morphism $\Phi: C^{\prime} \rightsquigarrow C$ of $\mathscr{C}$-coalgebras, 
two $\mathscr{P}$-algebras $A, A^{\prime}$, and an $\infty_{\alpha}$-morphism $\Psi: A \rightsquigarrow A^{\prime}$ which are such that the two composites

$$
\operatorname{hom}^{\alpha}(\Phi, 1) \operatorname{hom}^{\alpha}(1, \Psi) \text { and } \operatorname{hom}^{\alpha}(1, \Psi) \operatorname{hom}^{\alpha}(\Phi, 1)
$$

are not homotopic.

For simplicity, we will work in the non-symmetric setting. It is straightforward to construct a version of the example we present in the symmetric setting.

We take $\alpha: \mathrm{As}^{\vee} \rightarrow$ As to be the zero twisting morphism $\alpha=0$. This greatly simplifies the situation, because $\infty_{0}$-morphisms are very simple:

1. If $A$ is an associative algebra, then $\mathrm{B}_{0} A=\operatorname{As}^{\vee}(A)$ with the differential $d_{\mathrm{As}^{\vee}(A)}=$ $1_{\mathrm{As} \vee} \mathrm{o}^{\prime} d_{A}$ induced only by the differential of $A$. Therefore, an $\infty_{0}$-morphism of associative algebras $A \rightsquigarrow A^{\prime}$ is just a chain map $\operatorname{As}^{\vee}(A) \rightarrow A^{\prime}$.

2. Dually, an $\infty_{0}$-morphism of coassociative coalgebras $C^{\prime} \rightsquigarrow C$ is nothing else than a chain map $C^{\prime} \rightarrow \operatorname{As}(C)$.

Moreover, we will take our (co)algebras to be concentrated in degree 0 , and thus having trivial differential. Thus, we end up working with linear maps between vector spaces, and two such maps are homotopic if and only if they are equal.

The coassociative coalgebra $C^{\prime}$ will be

$$
C^{\prime}:=\mathbb{K} x \quad \text { with trivial coproduct, i.e. } \quad \Delta_{C^{\prime}}(x):=\mathrm{id} \otimes x \in \operatorname{As}^{\vee}\left(C^{\prime}\right) .
$$

For the coassociative coalgebra $C$ we take

$$
C:=\operatorname{As}^{\vee}(\mathbb{K} y)
$$

the cofree coassociative coalgebra over a 1 -dimensional vector space. The $\infty_{0}$-morphism $\Phi: C^{\prime} \rightsquigarrow C$ is given by the linear map

$$
\Phi: C^{\prime} \longrightarrow \operatorname{As}(C)
$$

defined by

$$
\Phi(x):=\mu_{2} \otimes\left(\left(\mu_{2}^{\vee} \otimes y \otimes y\right) \otimes(\mathrm{id} \otimes y)\right),
$$

where $\mu_{n} \in \operatorname{As}(n)$ is the operation corresponding to the multiplication of $n$ elements in an associative algebra.

For the algebras, we set $A$ to be

$$
A:=\operatorname{As}(\mathbb{K} z),
$$

the free associative algebra on one generator, and $A^{\prime}$ to be

$$
A^{\prime}:=\mathbb{K} w \quad \text { with } \quad \mu_{2}(w, w):=w .
$$

The $\infty_{0}$-morphism $\Psi: A \rightsquigarrow A^{\prime}$ is given by any linear map

$$
\Psi: \operatorname{As}^{\vee}(A) \longrightarrow A^{\prime}
$$

satisfying

$$
\Psi(\mathrm{id} \otimes(\mathrm{id} \otimes z))=w \quad \text { and } \quad \Psi\left(\mu_{2}^{\vee} \otimes((\mathrm{id} \otimes z) \otimes(\mathrm{id} \otimes z))\right)=w .
$$


For example, one can define $\Psi$ by the conditions above and setting it to be zero everywhere else.

Finally, we take $f \in \operatorname{hom}^{0}(C, A)$ to be any linear map such that $f($ id $\otimes y)=$ id $\otimes z$. For example, one can simply set $f$ to be 0 on a complement of $\mathbb{K}($ id $\otimes y)$ in $C$. We will consider the action of the two compositions on the element $\mu_{3}^{\vee} \otimes F:=\mu_{3}^{\vee} \otimes f \otimes f \otimes f$, and then apply the resulting map to $x \in C^{\prime}$. We refer to [RNW17, Sect. 6.1] for a diagrammatic description of the two composites. In formulæ, we have that

$$
\begin{aligned}
\operatorname{hom}^{0}(1, \Psi) \operatorname{hom}^{0}(\Phi, & 1)\left(\mu_{3}^{\vee} \otimes F\right)(x)=\Psi \operatorname{As}^{\vee}\left(\gamma_{A}\right) F^{\mathbb{S}} \operatorname{proj}_{3} \operatorname{As}^{\vee}(\Phi) \Delta_{C^{\prime}}(x) \\
= & \Psi \operatorname{As}^{\vee}\left(\gamma_{A}\right) F^{\mathbb{S}} \operatorname{proj}_{3} \operatorname{As}^{\vee}(\Phi)(\operatorname{id} \otimes x) \\
= & \Psi \operatorname{As}^{\vee}\left(\gamma_{A}\right) F^{\mathbb{S}} \operatorname{proj}_{3}\left(\operatorname{id} \otimes\left(\mu_{2} \otimes\left(\left(\mu_{2}^{\vee} \otimes y \otimes y\right) \otimes(\operatorname{id} \otimes y)\right)\right)\right) \\
= & 0,
\end{aligned}
$$

since the element in the second to last line lives in $\left(\mathrm{As}^{\vee} \circ \mathrm{As}\right)(2) \otimes C^{\otimes 2}$. At the same time, the other composition gives

$$
\begin{aligned}
& \operatorname{hom}^{0}(\Phi, 1) \operatorname{hom}^{0}(1, \Psi)\left(\mu_{3}^{\vee} \otimes F\right)(x)=\gamma_{A^{\prime}} \operatorname{As}(\Psi) F^{\mathbb{S}} \operatorname{proj}_{3} \operatorname{As}\left(\Delta_{C}\right) \Phi(x) \\
& =\gamma_{A^{\prime}} \operatorname{As}(\Psi) F^{\mathbb{S}} \operatorname{proj}_{3} \operatorname{As}\left(\Delta_{C}\right)\left(\mu_{2} \otimes\left(\left(\mu_{2}^{\vee} \otimes y \otimes y\right) \otimes(\mathrm{id} \otimes y)\right)\right) \\
& =\gamma_{A^{\prime}} \operatorname{As}(\Psi) F^{\mathbb{S}} \operatorname{proj}_{3}\left(\mu_{2} \otimes\left(\left(\left(\mathrm{id} \otimes\left(\mu_{2}^{\vee} \otimes y \otimes y\right)\right) \otimes(\mathrm{id} \otimes(\mathrm{id} \otimes y))\right)\right)\right. \\
& \left.+\left(\left(\mu_{2}^{\vee} \otimes((\mathrm{id} \otimes y) \otimes(\mathrm{id} \otimes y))\right) \otimes(\mathrm{id} \otimes(\mathrm{id} \otimes y))\right)\right) \\
& =\gamma_{A^{\prime}} \operatorname{As}(\Psi) F^{\mathbb{S}}\left(\mu_{2} \otimes\left(\left(\mu_{2}^{\vee} \otimes((\mathrm{id} \otimes y) \otimes(\mathrm{id} \otimes y))\right) \otimes(\mathrm{id} \otimes(\mathrm{id} \otimes y))\right)\right) \\
& =\gamma_{A^{\prime}} \operatorname{As}(\Psi)\left(\mu_{2} \otimes\left(\left(\mu_{2}^{\vee} \otimes((\mathrm{id} \otimes z) \otimes(\mathrm{id} \otimes z))\right) \otimes(\mathrm{id} \otimes(\mathrm{id} \otimes z))\right)\right) \\
& =\gamma_{A^{\prime}}\left(\mu_{2} \otimes w \otimes w\right) \\
& =w \text {. }
\end{aligned}
$$

Thus, the compositions are not equal, and, in particular, they are not homotopic.

\section{References}

[Ban17] R. Bandiera. Descent of Deligne-Getzler $\infty$-groupoids. 2017. arXiv: 1705.02880 .

[BM03] C. Berger and I. Moerdijk. Axiomatic homotopy theory for operads. Commentarii Mathematici Helvetici, 78(4):805-831, 2003. arXiv: math/0206094.

[DCH16] G. C. Drummond-Cole and J. Hirsh. Model structures for coalgebras. Proceedings of the AMS, 144(4):1467-1481, 2016. arXiv:1411.5526.

[DHR15] V. A. Dolgushev, A. E. Hoffnung, and C. L. Rogers. What do homotopy algebras form? Advances in Mathematics, 274:562-605, 2015. arXiv:1406.1751.

[Dol07] V. A. Dolgushev. Erratum to: "A proof of Tsygan's formality conjecture for an arbitrary smooth manifold". 2007. arXiv:math/0703113. 
[DP16] V. Dotsenko and N. Poncin. A tale of three homotopies. Applied Categorical Structures, 24(6):845-873, 2016. arXiv:1208.4695.

[DR15] V. A. Dolgushev and C. L. Rogers. A version of the Goldman-Millson theorem for filtered $L_{\infty}$-algebras. Journal of Algebra, 430:260-302, 2015. arXiv:1407.6735.

[Dup76] J. L. Dupont. Simplicial de Rham cohomology and characteristic classes of flat bundles. Topology, 15:233-245, 1976.

[Get09] E. Getzler. Lie theory for nilpotent $₫ \mathscr{L}_{\infty}$-algebras. Annals of Mathematics, 170(1):271-301, 2009. arXiv:math/0404003.

[Hin97] V. Hinich. Homological algebra of homotopy algebras. Communications in Algebra, 25(10):3291-3323, 1997. arXiv:q-alg/9702015.

[Hov99] M. Hovey. Model Categories, volume 63 of Mathematical Surveys and Monographs. AMS, 1999.

[Kon17] M. Kontsevich. Derived Grothendieck-Teichmüller group and graph complexes (after T. Willwacher). Report of the Séminaire Nicolas Bourbaki, 2016-2017(1126), 2017.

[LG16] B. Le Grignou. Homotopy theory of unital algebras. 2016. arXiv: 1612.02254 .

[LV12] J. L. Loday and B. Vallette. Algebraic Operads, volume 346 of Grundlehren der Mathematischen Wissenschaften. Springer Verlag, 2012.

[RN17] D. Robert-Nicoud. Representing the deformation $\infty$-groupoid. 2017. To appear in Algebr. Geom. Topol. arXiv:1702.02529.

[RN18a] D. Robert-Nicoud. Deformation theory with homotopy algebra structures on tensor products. Documenta Mathematica, 23:189-240, 2018. arXiv:1702.02194.

[RN18b] D. Robert-Nicoud. A model structure for the Goldman-Millson theorem. Graduate Journal of Mathematics, 3(1):15-30, 2018. arXiv:1803.03144.

[RNW17] D. Robert-Nicoud and F. Wierstra. Homotopy morphisms between convolution homotopy Lie algebras. 2017. To appear in J. Noncommut. Geom. arXiv:1712.00794.

[Val14] B. Vallette. Homotopy theory of homotopy algebras. 2014. arXiv: 1411.5533.

[Wie16] F. Wierstra. Algebraic Hopf invariants and rational models for mapping spaces. 2016. arXiv:1612.07762.

Daniel Robert-Nicoud robert-nicoud@math.univ-paris13.fr

Laboratoire Analyse, Géométrie et Applications, Université Paris 13, 99 Avenue Jean Baptiste Clément, 93430 Villetaneuse, France

Felix Wierstra felix.wierstra@gmail.com

Faculty of Mathematics and Physics, Charles University, Sokolovská 49/83, 18675 Praha 8, Czech Republic 